Computational Mechanics (1992) 10,73-95

Computational Mechanics

(C) Sprnger-Verlag 1992

\title{
A comparison of homogenization and standard mechanics analyses for periodic porous composites
}

\author{
S. J. Hollister ${ }^{1}$ and N. Kikuchi ${ }^{2}$ \\ ${ }^{1}$ Orthopaedic Research Laboratories, Section of Orthopaedic Surgery, Rm. G-0161, 400 N. Ingalls Bldg., The University of \\ Michigan, Ann Arbor, MI 48109, USA \\ ${ }^{2}$ Computational Mechanics Laboratory, Department of Mechanical Engineering and Applied Mechanics, The University of \\ Michigan, Ann Arbor, MI 48109, USA
}

\begin{abstract}
Composite material elastic behavior has been studied using many approaches, all of which are based on the concept of a Representative Volume Element (RVE). Most methods accurately estimate effective elastic properties when the ratio of the RVE size to the global structural dimensions, denoted here as $\eta$, goes to zero. However, many composites are locally periodic with finite $\eta$. The purpose of this paper was to compare homogenization and standard mechanics RVE based analyses for periodic porous composites with finite $\eta$. Both methods were implemented using a displacement based finite element formulation. For one-dimensional analyses of composite bars the two methods were equivalent. However, for two- and three-dimensional analyses the methods were quite different due to the fact that the local RVE stress and strain state was not determined uniquely by the applied boundary conditions. For two-dimensional analyses of porous periodic composites the effective material properties predicted by standard mechanics approaches using multiple cell RVEs converged to the homogenization predictions using one cell. In addition, homogenization estimates of local strain energy density were within $30 \%$ of direct analyses while standard mechanics approaches generally differed from direct analyses by more than $70 \%$. These results suggest that homogenization theory is preferable over standard mechanics of materials approaches for periodic composites even when the material is only locally periodic and $\eta$ is finite.
\end{abstract}

\section{Introduction}

Composite materials have been used extensively in engineering applications due to their high strength to weight ratios. Natural materials, including wood and human bone tissue, are also composite materials with complex microstructures optimized for withstanding functional loads. For example, the low mass composite structures of bone tissue enable an organism to move efficiently and withstand high structural loads while minimizing metabolic costs. The prevalence of composite structures in nature along with their increasing engineering applications suggest that these materials will become the rule rather than the exception in structural design and analysis. Increased application of composite materials necessitates accurate yet feasible methods for analyzing composite material mechanics.

Composite materials are frequently used to fabricate large structural components. Yet the behavior of these components depends on the composite microstructure. Analyzing large structures on a microstructural level, however, is clearly an intractable problem. Analysis methods have therefore sought to approximate composite structural mechanics by analyzing a representative section of the composite microstructure, commonly called a Representative Volume Element (RVE). The term RVE seems to have been coined by Hill (1963) and has also been detailed extensively by Hashin and co-workers (reviewed in Hashin 1983).

RVE based methods decouple analysis of a composite material into analyses at the local and global levels. The local level analysis models the microstructural details to determine effective elastic properties. The local level analysis can also be used to calculate the relationship of the effective or average RVE strain to the local strain within the RVE. The composite structure is then replaced by an equivalent homogeneous material having the calculated effective properties. The global level analysis calculates the effective or average stress and strain within the equivalent homogeneous structure. The process of calculating effective properties has been termed "homogenization" by Suquet (1987). If local stress and strain estimates are needed, they can be computed 
using the relationship between the average and local strain obtained from the local analysis. This procedure has been termed "localization" (Suquet 1987).

There are a number of RVE approaches to analyze composite materials, each of which may give different results depending upon the assumptions made and the ratio of the RVE size to the size of the global region of interest, denoted here as $\eta$. In general, the accuracy of RVE approaches increases as $\eta \rightarrow 0$. For many composite materials, however, $\eta$ is fixed and significantly larger than 0 . For these cases, it is important to know how choice of analysis method and RVE size will affect the accuracy of the analysis. Ideally, one would like to obtain the maximum accuracy for the smallest RVE size. This paper compares homogenization theory to a finite element implementation of standard mechanics of materials approaches for the analysis of periodic porous composites with $\eta$ equal to 0.2 . The accuracy of effective stiffness estimates was assessed by comparing the predictions of the two approaches directly. The accuracy of local strain energy density estimates was assessed by comparing the estimates of each RVE approach separately to a direct analysis of the whole composite material. It was shown that homogenization theory gave more accurate estimates of effective stiffness and local strain energy than standard mechanics of materials approaches for periodic porous composites.

\section{Review of RVE based composite analysis methods}

Effective material property identification for composite materials dates back to the $19^{\text {th }}$ century, including the famous Voigt (1889) and Reuss (1929) bounds. One of the first formal definitions of the RVE was given by Hill (1963) who stated that the RVE was 1) structurally entirely typical of the composite material on average and 2) contained a sufficient number of inclusions such that the apparent moduli were independent of the RVE boundary displacements or tractions. Hashin (1983) also emphasized the nature of RVE analysis stating that stress and strain fields in the RVE should be statistically homogeneous when subjected to homogeneous boundary conditions except in a layer near the external surface.

The statements by Hill and Hashin reflect the crux of RVE based analysis methods. Since it is generally impossible to analyze an entire composite structure, RVE analyses predict the effective stiffness and the relationship of average to local strain for the RVE and assume that these quantities represent the entire composite structure. This is done by applying boundary conditions to the RVE and solving the resultant boundary value problem. The applied boundary conditions, however, cannot represent all the possible in-situ boundary conditions to which the RVE is subjected within the composite. The accuracy of the RVE approximation depends on how well the assumed boundary conditions reflect each of the myriad boundary conditions to which the RVE is subjected in-situ.

Consider the case where the in-situ boundary conditions differ from the applied boundary conditions, but produce the same average RVE strain. In this case the average stiffness predicted by the RVE analysis must be greater than the actual stiffness by the principle of minimum strain energy. The in-situ boundary conditions would minimize the energy while the assumed boundary conditions would be admissible and by definition produce greater energy. The average stress within the RVE under assumed boundary conditions must be higher to produce a higher energy. The same argument holds for applied tractions boundary conditions with the principle of minimum complementary energy. In this situation, the homogeneous traction boundary condition will produce a higher complementary energy than an in-situ traction condition for the same average stress giving a higher compliance and therefore a lower stiffness. Thus, RVE analyses under applied displacements give an upper bound on apparent stiffness while applied tractions give a lower bound.

The difference between average stress states under admissible and actual boundary displacements is greatest when $\eta$ is large. The same is true for average strain states under admissible and actual boundary tractions. Conversely, in standard mechanics approaches, boundary layer effects are most prevalent when the RVE is small (Hill 1963). These boundary layer effects decrease when the RVE size is increased in accordance with St. Venant's principle (Fung 1965). These two results 
create a paradox for a fixed size global analysis region since increasing the RVE size also increases $\eta$. Moreover, larger RVEs increase the complexity of the resulting boundary value problem. Therefore, it is desirable to choose a method that gives the most accuracy for the smallest RVE, which for periodic materials is the smallest repeating unit.

\subsection{Mechanics of materials approaches for porous cellular materials}

A number of investigators have used either analytical or finite element based mechanics of materials approaches to calculate the effective elastic properties of porous cellular materials (Beaupre and Hayes 1985, Christenson 1986, Gibson and Ashby 1982, Gibson and Ashby 1988, Huber and Gibson 1988, Iremonger and Lawler 1980, Ishai and Cohen 1967, Kanakkanatt 1973, Ko 1965, Lederman 1971, Patel and Finnie 1970, Warren and Kraynik 1988). In the analytical approaches, uniform boundary tractions or displacements on the RVE boundary are assumed to cause either bending or axial compression of the cell walls. The average stress induced by the boundary tractions is divided by the local strain calculated using beam or plate solutions to give the apparent modulus. For beam bending, it can be shown (Gibson and Ashby 1982) that the apparent modulus $\bar{E}$ is related to the modulus of the cell wall material $E$ by

$\bar{E}=C E\left(\frac{\rho}{\rho_{s}}\right)^{2}$,

where $C$ is a proportionality constant, $\rho$ is the apparent density of the material, and $\rho_{s}$ is the density of the cell wall material. For axial compression the relationship between the apparent and cell wall moduli is (Christenson 1986, Gibson and Ashby 1988)

$\bar{E}=C E\left(\frac{\rho}{\rho_{s}}\right)$,

where the terms are the same as defined in Eq. 2.1.

Mechanics of materials approaches often provide direct insight into the relationship between effective stiffness and microstructural geometry. However, the difference between apparent moduli calculated under different assumptions can be quite large. For a material with relative density of 0.1, the apparent stiffness estimate of Eq. 2.2 is 10 times the apparent stiffness estimate of Eq. 2.1 . Another difficulty is the assumption that traction boundary conditions directly translate into uniform loads within the cell wall. Errors in this assumption were demonstrated by Beaupre and Hayes (1985) in a finite element study of a two-dimensional porous material consisting of four repeated unit cells. They found that the boundary displacements on the inner unit cells had a nonlinear shape despite application of uniform linear boundary displacements on the edges of the outer unit cells. The stiffness calculated using a linear boundary displacement was 7.5 times higher than the stiffness calculated using a nonlinear boundary displacement on the RVE. These results are consistent with the theoretical basis of stiffness bounds discussed earlier and illustrate the sensitivity of effective stiffness predictions to RVE boundary condition assumptions.

\subsection{Self-consistent methods}

Self-consistent methods, which encompass a number of related approaches, have been frequently applied to estimate effective elastic properties. Self-consistent methods analyze the composite material as an intially homogeneous material or matrix into which inclusions of a different material have been placed. The total strain $\varepsilon_{i j}$ in the material at any point is equal to the strain in the homogeneous material $\varepsilon_{k l}^{0}$ plus the deviation in strain $\varepsilon_{k l}^{d}$ due to the presence of the inclusions. The basis of the self-consistent methods is the introduction of a transformation strain $\varepsilon_{k l}^{*}$ such that the stress within the inclusion is equal to the stress within the matrix modified by the polarization 
stress (Nemat-Nasser et al. 1982, Benveniste 1987):

$C_{i j k l}^{m}\left(\varepsilon_{k l}^{0}+\varepsilon_{k l}^{d}+\varepsilon_{k l}^{*}\right)=C_{i j k l}^{i}\left(\varepsilon_{k l}^{0}+\varepsilon_{k l}^{d}\right)$,

where $C_{i j k l}^{m}$ is the matrix stiffness, $C_{i j k l}^{i}$ is the inclusion stiffness, $\varepsilon_{k l}^{0}$ is the strain in the homogeneous

matrix, $\varepsilon_{k l}^{d}$ is the perturbation strain, $\varepsilon_{k l}^{*}$ is the transformation strain, and the polarization stress is given by $C_{i j k l}^{m} \varepsilon_{k l}^{*}$. The task is then to estimate the perturbation strain based on an assumption about the nature of the transformation strain.

The most common assumption is that the transformation strain is constant within the inclusion, based on the classic work of Eshelby (1957). Hashin and Shtrikman (1962, 1963, reviewed by Hashin 1983) used the concept of polarization stress within variational principles to derive bounds for macroscopically isotropic composites with spherical inclusions. Hill (1965) and Budiansky (1965) derived bounds within Hashin and Shtrikman's for the same type of composites using Eshelby's solution. The bounds derived using Eshelby's solution are closest when the relative stiffness of the phases is less than 10 and the concentration of the inclusions is dilute. In fact, the lower bounds derived by Hashin and Shtrikman, Hill, and Budiansky equal zero for a porous composite with a volume fraction of voids greater than 0.5 (Hashin 1983).

A significant amount of work has been done to improve the self-consistent estimates of effective material properties (Accorsi 1988, Benveniste 1987, Cleary et al. 1980, Kroner 1977, Mori and Tanaka 1973, Mura 1982, Norris 1985, 1989, Walpole 1966a,b, Willis 1977). Of particular relevance to periodic porous composite analysis is the extensive work of Nemat-Nasser and co-workers (Nemat-Nasser and Taya 1981, Nemat-Nasser et al. 1982, Iwakuma and NematNasser 1983, Accorsi and Nemat-Nasser 1986) who introduced Fourier series expansions to approximate the periodic fluctuations of the transformation strain and the deviation fields of displacement, strain, and stress resulting from periodic geometry. A significant outcome of this approach is that Eshelby's tensor, which relates the inclusion strain in the absence of the surrounding matrix to the inclusion strain with the surrounding matrix, is not constant as in classic self-consistent solutions. Instead, Eshelby's tensor is a periodically fluctuating function. NematNasser et al. (1982) found that this approach very accurately estimated the effective stiffness of porous glass composites. They commented that assuming periodically varying fields successfully accounted for the interaction between inclusions at non dilute concentrations even when the material was not periodic.

\subsection{Homogenization theory}

Homogenization theory developed from studies of partial differential equations with rapidly varying coefficients. Two explicit assumptions are made in homogenization theory. First, it is assumed that fields vary on multiple spatial scales due to the existence of a microstructure. Second, it is assumed that the microstructure is spatially periodic. The relevant field variables are approximated by an asymptotic expansion

$u_{i}^{\eta}\left(x_{i}, y_{i}\right)=u_{0 i}\left(x_{i}, y_{i}\right)+\eta u_{1 i}\left(x_{i}, y_{i}\right)+\eta^{2} u_{2 i}\left(x_{i}, y_{i}\right)+\cdots$,

where $u_{i}^{\eta}$ is the exact value of the field variable, $u_{0 i}$ is the macroscopic or average value of the field variable, $u_{1 i}, u_{2 i}$, etc. are perturbations in the field variables due to the microstructure, $x_{i}$ are the global level coordinates, $y_{i}$ are the micro level coordinates, and $\eta$ is the ratio of the microstructure size to the total size of the analysis region. In elasticity theory $u_{0 i}$ would be the continuum level displacements while $u_{1 i}$ would be the microstructural displacements. The macro coordinates $x_{i}$ are related to the micro coordinates $y_{i}$ by

$y_{i}=\frac{x_{i}}{\eta}$

Equation (2.5) implies that quantities on the local level like stress vary $1 / \eta$ times more rapidly than corresponding global level quantities. This asymptotic expansion is substituted into the governing 
differential equations and terms of order $O(\eta)$ are separated and set equal to zero. The resulting equations govern the mechanical behavior of the composite material at different levels of structure.

The earliest use of homogenization theory in the West can be attributed to Babuska (1976a-d), Keller (1977), Larsen (1975) and Sanchez-Palencia (1974). Independent work on homogenization analysis was also done in the Soviet Union beginning in the early 1970's. Bakhvalov and Panasenko (1989) summarize and reference much of the Soviet work in their extensive text on homogenization theory. Texts on mathematical aspects and engineering applications of homogenization theory to many diverse fields have been written by Bensoussan et al. (1978).

In early studies, Babuska discussed many mathematical aspects of homogenization theory as applied to elliptic partial differential equations including the use of boundary layer analysis and extensions to nonlinear cases. Babuska and others (see Bakhvalov and Panasenko 1989, SanchezPalencia 1980) noted that the macroscopic displacement from the asymptotic expansion would converge to the average displacement of the actual material as

$\left\|u_{0 i}-u_{i}^{\eta}\right\|_{L_{2}} \leqq C \eta^{p}$

where the norm is defined in the space of square integrable functions $L_{2}, p$ denotes the order of terms retained from the expansion, and $C$ is a constant.

Other studies on the accuracy of homogenization theory have used the concept of Generalized or $G$-convergence (Zhikov et al. 1979). The premise of $G$-convergence is that the true differential operator field variable product will converge to the homogenized differential operator field variable product in the limit $\eta \rightarrow 0$. For the elasticity problem, $G$-convergence states

$\lim _{\eta \rightarrow 0} \frac{\partial}{\partial x_{i}}\left[C_{i j k l}^{\eta} \frac{\partial u_{k}^{\eta}}{\partial x_{l}}\right] \rightarrow \frac{\partial}{\partial x_{i}}\left[\bar{C}_{i j k l} \frac{\partial u_{0 k}}{\partial x_{l}}\right]$.

The estimate (2.7) shows the mechanical behavior of the homogenized material more closely represents that of the true composite material as the size of the microstructure relative to the global body size decreases.

Extensive application of homogenization theory to study composite materials began in France in the late 1970's and continued through the 1980's (Bensoussan et al. 1978, Dumontet 1985, Duvaut 1976, Lene and Leguillon 1982, Lions 1980, 1981, Sanchez-Palencia 1980, 1987, Suquet 1987). Lions studied convergence of elliptic operators specifically for porous media and found the same convergence estimate as (2.6). Suquet (1987) studied solutions of the microscopic equilibrium equation derived from (2.4) with different applied boundary conditions. He found that applied displacements overestimated and applied tractions underestimated the effective stiffness of metal sheets with small perforations. The use of periodic boundary conditions with the homogenization formulation gave the closest agreement with experimental results. Other researchers who have applied homogenization theory to study composite materials include Murakami and colleagues (Murakami et al. 1981, Murakami and Toledano 1990) who studied laminated composites and Guedes and Kikuchi (1990) who investigated computational aspects of homogenization theory as applied to composite material analysis.

The convergence estimates (2.6) provided by Babuska, Bakhvalov and Panasenko, Lions, and others again emphasize that accuracy of the homogenization analysis depends on $\eta$. The results of Suquet (1987) show that the assumption of periodic boundary conditions is also important for obtaining accurate results. The size of the error in (2.6) is partially determined by the constant $C$. $C$ itself may depend on the relative stiffness and geometry of the composite constituents as well as the boundary conditions applied to the global analysis region. Furthermore, (2.6) gives error bounds for the difference in displacements integrated over the RVE volume but does not provide information on the distribution of error within the RVE. Information on the error distribution within the RVE may be important for studies concerned with localized stress and strain states of the composite microstructure.

Although detailed error bounds are available for homogenization analysis, it appears that no such bounds are available for standard mechanics of materials approaches. For such approaches, it is known that accuracy in predicting effective stiffness depends on the RVE size compared to 
the global analysis region (Hill 1963). However, similar to homogenization theory, the effect of RVE to global analysis region size and RVE boundary condition assumptions on the local error distribution is not known. Furthermore, unlike homogenization theory, effective stiffness predictions will change as the number of repeating units contained in the RVE changes.

\section{Theoretical background}

\subsection{Standard mechanics approach}

In the standard mechanics approach a chosen RVE is generally analyzed using either uniform traction or uniform displacement boundary conditions. These boundary conditions are chosen so as to produce an average strain (if displacements are used) or an average stress (if tractions are applied) within a homogeneous material of the same size as the RVE. The relationship between the average strain and the displacement boundary conditions can be written using the divergence theorem as

$\bar{\varepsilon}_{i j}=\frac{1}{\left|V_{\mathrm{RVE}}\right|} \int_{V_{\mathrm{RVE}}} \varepsilon_{i j} d V_{\mathrm{RVE}}=\frac{1}{\left|V_{\mathrm{RVE}}\right|} \int_{S_{\mathrm{RVE}}} \frac{1}{2}\left(u_{i} n_{j}+u_{j} n_{i}\right) d S_{\mathrm{RVE}}$

where $\bar{\varepsilon}_{i j}$ is the average strain, $\varepsilon_{i j}$ is the local strain in the RVE, $u_{i}$ is the displacement imposed on the RVE boundary, $n_{i}$ is the normal vector to the RVE boundary, and $S_{\mathrm{RVE}}$ is the RVE boundary. The relationship between the average stress and the boundary tractions can be written as (Hill 1963)

$\bar{\sigma}_{i j}=\frac{1}{\left|V_{\mathrm{RVE}}\right|} \int_{V_{\mathrm{RVE}}} \sigma_{i j} d V_{\mathrm{RVE}}=\frac{1}{\left|V_{\mathrm{RVE}}\right|} \int_{S_{\mathrm{RVE}}} \frac{1}{2}\left(t_{i} y_{j}+t_{j} y_{i}\right) d S_{\mathrm{RVE}}$,

where $\bar{\sigma}_{i j}$ is the average stress, $\sigma_{i j}$ is the local stress in the RVE, $t_{i}$ is the traction imposed on the RVE boundary, $y_{i}$ are local coordinates of the RVE boundary, and $S_{\mathrm{RVE}}$ is the RVE boundary. It is important to note that there is no unique relationship between the average stress or strain and the boundary tractions or displacements in the two- or three-dimensional case. In other words, a number of different boundary displacements integrated over the boundary may produce the same average strain. Since the in-situ boundary conditions are not known, the displacements in (3.1) or the tractions in (3.2) are generally chosen to be uniform (Fig. 3.1).

Once the boundary conditions are chosen, the standard weak form of the equilibrium equations is solved to calculate the local RVE strain. The weak form of the RVE equilibrium equations for

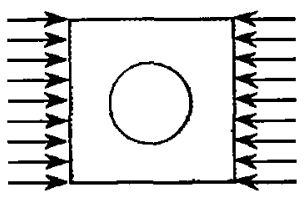

y1 normal

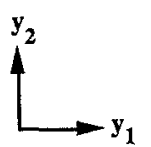

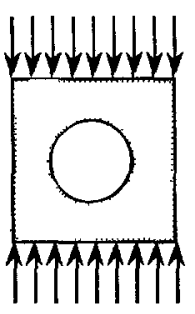

y2 normal

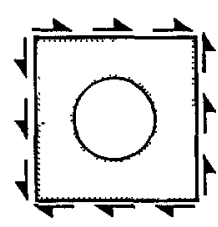

y1 $_{2}$ shear
Fig. 3.1. Uniform boundary conditions applied to the RVE in both the applied displacement and applied traction approach. The boundary conditions will produce $x$ normal, $y$ normal, and $x y$ shear stresses (applied traction) or strains (applied displacements) in a homogeneous material of the same size as the RVE 
the case of applied boundary tractions is solved once for applied stress states corresponding to each component $k l$ of the stress tensor

$\int_{V_{\mathrm{RVE}}} C_{i j m n} \varepsilon_{i j}(v) \varepsilon_{m n}^{k l}(u) d V_{\mathrm{RVE}}=\int_{S_{\mathrm{RVE}}} t_{i}^{k l} v_{i} d S_{\mathrm{RVE}}$,

where $t^{k l}$ is the boundary traction which would produce an average stress $\bar{\sigma}_{k l}$ in a homogeneous material, $C_{i j m n}$ is the stiffness tensor of the RVE material components, $\varepsilon_{i j}(v)$ is the virtual strain, $\varepsilon_{m n}^{k l}(u)$ is the total microstructural strain for the $k l^{\text {th }}$ traction, and $v_{i}$ is the virtual displacement. Due to symmetry of the stress tensor, (3.3) need only be solved three times for two-dimensional problems and six times for three-dimensional problems. The weak form of the RVE equilibrium equations for the case of applied boundary displacements is

$\int_{V_{\mathrm{RVE}}} C_{i j m n} \varepsilon_{i j}(v) \varepsilon_{m n}^{k l}(u) d V_{\mathrm{RVE}}=\int_{S_{\mathrm{RVE}}} \lambda v_{i}\left(u_{i}^{k l}-g_{i}^{k l}\right) d S_{\mathrm{RVE}}$

where the boundary displacements are implemented using a penalty method and $\lambda$ is the penalty parameter, $u_{i}^{k l}$ is the boundary displacement, $g_{i}^{k l}$ is the specified displacement which would produce a uniform average strain $\bar{\varepsilon}_{k l}$ in a homogeneous material, and the other terms are as defined previously. Once the three strain states (six for 3-D) using (3.3) or (3.4) are determined, the local structure tensor $M_{i j p m}$ which relates the average strain $\bar{\varepsilon}_{p m}^{k l}$ and the local or microstructural total strain $\varepsilon_{i j}^{k l}$ may be calculated from

$\varepsilon_{i j}^{k l}=M_{i j p m} \bar{\varepsilon}_{p m}^{k l}$.

The local structure tensor $M_{i j p m}$ has minor symmetries such that $M_{i j p m}=M_{j i p m}=M_{i j m p}$, but in general does not have major symmetry $M_{i j p m} \neq M_{p m i j}$. Once $M_{i j k l}$ is determined, the local strain at any point within the RVE may be calculated from an arbitrary average strain as

$\varepsilon_{i j}=M_{i j k l} \bar{\varepsilon}_{k l}$.

The effective stiffness tensor $\bar{C}_{i j k l}$ which relates the average strain to the average stress

$\bar{\sigma}_{i j}=\bar{C}_{i j k l} \bar{\varepsilon}_{k l}$

may also be calculated from $M_{i j k l}$. Starting from Hooke's law at the microscopic level

$\sigma_{i j}=C_{i j k l} \varepsilon_{k l}$,

both sides are integrated over the RVE and divided by the total RVE volume to give

$\frac{1}{\left|V_{\mathrm{RVE}}\right|} \int_{V_{\mathrm{RVE}}} \sigma_{i j} d V_{\mathrm{RVE}}=\frac{1}{\left|V_{\mathrm{RVE}}\right|} \int_{V_{\mathrm{RVE}}} C_{i j k l} \varepsilon_{k l} d V_{\mathrm{RVE}}$.

Substituting for $\varepsilon_{k l}$ from (3.6) and recalling (3.1) and (3.2) gives

$\bar{\sigma}_{i j}=\frac{1}{\left|V_{\mathrm{RVE}}\right|} \int_{V_{\mathrm{RVE}}} C_{i j p m} M_{p m k l} d V_{\mathrm{RVE}} \bar{\varepsilon}_{k l}$,

from which the effective stiffness tensor may be defined as

$\bar{C}_{i j k l}=\frac{1}{\left|V_{\mathrm{RVE}}\right|} \int_{V_{\mathrm{RVE}}} C_{i j p m} M_{p m k l} d V_{\mathrm{RVE}}$

\subsection{Homogenization theory}

In homogenization theory, the microscopic coordinates $y$ and the macroscopic coordinates $\boldsymbol{x}$ are related by

$y_{i}=\frac{x_{i}}{\eta}$ 
where $\eta$ is the ratio between the RVE size and the size of the macroscopic region in which it exists. Physically (3.8) means that quantities like stress or strain will vary $\frac{1}{\eta}$ faster on the microscopic level than on the macroscopic level. Derivatives of any function $g^{\eta}(\boldsymbol{x})=g\left(\boldsymbol{x}, \boldsymbol{y}=\frac{\boldsymbol{x}}{\eta}\right)$ with respect to
$\boldsymbol{x}$ are written using the chain rule and (3.8) as $\frac{\partial g^{\eta}}{\partial x_{i}}=\frac{\partial g}{\partial x_{i}}+\frac{1}{\eta} \frac{\partial g}{\partial y_{i}}$.

Applying (3.9) to the asymptotic expansion of the displacement $\boldsymbol{u}$ in (2.4) allows the small deformation strain tensor to be written as

$\varepsilon_{i j}(\boldsymbol{u})=\frac{1}{2}\left(\frac{\partial u_{i}^{\eta}}{\partial x_{j}}+\frac{\partial u_{j}^{\eta}}{\partial x_{i}}\right)=\frac{1}{2}\left[\left(\frac{\partial u_{0 i}}{\partial x_{j}}+\frac{\partial u_{0 j}}{\partial x_{i}}\right)+\left(\frac{\partial u_{1 i}}{\partial y_{j}}+\frac{\partial u_{1 j}}{\partial y_{i}}\right)+\eta\left(\frac{\partial u_{1 i}}{\partial x_{j}}+\frac{\partial u_{1 j}}{\partial x_{i}}\right)\right]+$ h.o.t.

where h.o.t. denotes higher order terms and the other terms are as defined previously. The following strain tensors can be defined from (3.10) (neglecting terms of $O(\eta)$ and higher)

$\varepsilon_{i j}=\bar{\varepsilon}_{i j}+\varepsilon_{i j}^{*}, \quad \bar{\varepsilon}_{i j}=\frac{1}{2}\left(\frac{\partial u_{0 i}}{\partial x_{j}}+\frac{\partial u_{0 j}}{\partial x_{i}}\right), \quad \varepsilon_{i j}^{*}=\frac{1}{2}\left(\frac{\partial u_{1 i}}{\partial y_{j}}+\frac{\partial u_{1 j}}{\partial y_{i}}\right)$,

where $\varepsilon_{i j}$ is the local or microstructural strain tensor, $\bar{\varepsilon}_{i j}$ is the average or macroscopic strain tensor as defined previously and $\varepsilon_{i j}^{*}$ has been denoted as the fluctuating strain tensor by Suquet (1987). The fluctuating strain tensor is assumed to vary periodically. The higher order terms may be included to improve the accuracy of the analysis, but require solving additional equations (Bourgat 1977). For the virtual displacement or weak form of the equilibrium equations, the virtual displacement $v$ and hence the virtual strain $\varepsilon_{i j}(v)$ is also expanded asymptotically as a function of $\boldsymbol{x}$ and $\boldsymbol{y}$

$\varepsilon_{i j}(v)=\frac{1}{2}\left(\frac{\partial v_{i}}{\partial x_{j}}+\frac{\partial v_{j}}{\partial x_{i}}\right)=\frac{1}{2}\left[\left(\frac{\partial v_{0 i}}{\partial x_{j}}+\frac{\partial v_{0 j}}{\partial x_{i}}\right)+\left(\frac{\partial v_{1 i}}{\partial y_{j}}+\frac{\partial v_{1 j}}{\partial y_{i}}\right)+\eta\left(\frac{\partial v_{1 i}}{\partial x_{j}}+\frac{\partial v_{1 j}}{\partial x_{i}}\right)\right]+$ h.o.t.

After neglecting terms of order $O(\eta)$ and higher, the following definitions of the virtual strain components are made for convenience

$\varepsilon_{i j}(v)=\varepsilon_{i j}^{0}(v)+\varepsilon_{i j}^{1}(v), \quad \varepsilon_{i j}^{0}(v)=\frac{1}{2}\left(\frac{\partial v_{0 i}}{\partial x_{j}}+\frac{\partial v_{0 j}}{\partial x_{i}}\right), \quad \varepsilon_{i j}^{1}(v)=\frac{1}{2}\left(\frac{\partial v_{1 i}}{\partial y_{j}}+\frac{\partial v_{1 j}}{\partial y_{i}}\right)$.

The expanded forms of the strain tensors (3.11a and 3.13a) are substituted into the standard weak form of the equilibrium equations given by

$$
\int_{\Omega^{\eta}} C_{i j k l} \varepsilon_{i j}(v) \varepsilon_{k l}(u) d \Omega^{\eta}=\int_{\Gamma} t_{i} v_{i} d \Gamma,
$$

where here it is assumed that $\Omega^{\eta}$ represents the total macroscopic plus microscopic domain of the composite material and the tractions $t_{i}$ (as well as any boundary displacements) are applied only to the macroscopic boundaries of the composite, not to any interior boundaries in the microstructure. The substitution of the expanded strain tensors into (3.14) yields

$$
\int_{\Omega^{\eta}} C_{i j k l}\left(\varepsilon_{i j}^{0}(v)+\varepsilon_{i j}^{1}(v)\right)\left(\bar{\varepsilon}_{k l}+\varepsilon_{k l}^{*}\right) d \Omega^{\eta}=\int_{\Gamma} t_{i} v_{i} d \Gamma \text {. }
$$

Since $v$ is an arbitrary function, it may be chosen to vary only on the macroscopic or microscopic level. If $v$ varies only on the microscopic level and is constant on the macroscopic level (giving $\left.\varepsilon_{i j}^{0}(v)=0\right)$, then the microscopic equilibrium equation is obtained

$\int_{\Omega^{\eta}} C_{i j k l} \varepsilon_{i j}^{1}(v)\left(\bar{\varepsilon}_{k l}+\varepsilon_{k l}^{*}\right) d \Omega^{\eta}=0$. 
If $v$ varies only on the macroscopic level and is constant on the microscopic level (giving $\varepsilon_{i j}^{1}(v)=0$ ), the macroscopic equilibrium equation is obtained

$$
\int_{\Omega^{n}} C_{i j k l} \varepsilon_{i j}^{0}(v)\left(\bar{\varepsilon}_{k l}+\varepsilon_{k l}^{*}\right) d \Omega^{\eta}=\int_{\Gamma} t_{i} v_{i} d \Gamma \text {. }
$$

Since $\varepsilon_{k l}^{*}$ varies periodically, (3.16a) and (3.16b) may be rewritten assuming $\eta$ in the limit goes to zero as

$\int_{\Omega} \frac{1}{\left|V_{\mathrm{RVE}}\right|} \int_{V_{\mathrm{RVE}}} C_{i j k l} \varepsilon_{i j}^{1}(v)\left(\bar{\varepsilon}_{k l}+\varepsilon_{k l}^{*}\right) d V_{\mathrm{RVE}} d \Omega=0$

$\int_{\Omega} \frac{1}{\left|V_{\mathrm{RVE}}\right|} \int_{V_{\mathrm{RVE}}} C_{i j k l} \varepsilon_{i j}^{0}(v)\left(\bar{\varepsilon}_{k l}+\varepsilon_{k l}^{*}\right) d V_{\mathrm{RVE}} d \Omega=\int_{\Gamma} t_{i} v_{i} d \Gamma$.

Equation (3.17a) will be satisfied if the integral over the RVE is zero. This means (3.17a) may be rewritten as

$\int_{V_{\mathrm{RVE}}} C_{i j k l} \varepsilon_{i j}^{1}(v) \varepsilon_{k l}^{*} d V_{\mathrm{RVE}}=-\int_{V_{\mathrm{RVE}}} C_{i j k l} \varepsilon_{i j}^{1}(v) \bar{\varepsilon}_{k l} d V_{\mathrm{RVE}}$.

In general $\bar{\varepsilon}_{k l}$ is not known a priori. However, since the problem is linear, any arbitrary $\bar{\varepsilon}_{k l}$ may be written as a linear combination of unit strains which will be defined for the two-dimensional case as (similar to Suquet 1987)

$\bar{\varepsilon}_{p m}^{11}=-\left[\begin{array}{ll}1 & 0 \\ 0 & 0\end{array}\right], \quad \bar{\varepsilon}_{p m}^{22}=-\left[\begin{array}{ll}0 & 0 \\ 0 & 1\end{array}\right], \quad \bar{\varepsilon}_{p m}^{12}=-\left[\begin{array}{ll}0 & 1 \\ 0 & 0\end{array}\right], \quad \bar{\varepsilon}_{p m}^{21}=-\left[\begin{array}{ll}0 & 0 \\ 1 & 0\end{array}\right]$.

Substituting the unit strains into the right hand side of (3.18) gives a stress tensor $\sigma_{i j}^{* k l}=C_{i j p m} \bar{\varepsilon}_{p m}^{k l}$.

An auxiliary equation to solve for $\varepsilon_{p m}^{*}$ in (3.18) using (3.19) is

$\int_{V_{\mathrm{RVE}}} C_{i j p m} \varepsilon_{i j}^{1}(v) \varepsilon_{p m}^{* k l}(u) d V_{\mathrm{RVE}}=\int_{V_{\mathrm{RVE}}} \varepsilon_{i j}^{1}(v) \sigma_{i j}^{* k l} d V_{\mathrm{RVE}}$.

To ensure periodicity of the strain field $\varepsilon_{i j}^{* k l}$, the displacements at opposite sides of the RVE are constrained to be equal.

Once $\varepsilon_{i j}^{* k l}$ is determined, the solution to (3.18) may be recovered by $\varepsilon_{i j}^{*}=-\varepsilon_{i j}^{* k l} \bar{\varepsilon}_{k l}$.

Recalling (3.11a), the relationship between the local RVE strain and the average strain may be written using the local structure tensor as

$\varepsilon_{i j}=M_{i j k l} \overline{\bar{c}}_{k l}, \quad M_{i j k l}=\frac{1}{2}\left(\delta_{i k} \delta_{j l}+\delta_{i l} \delta_{j k}\right)-\varepsilon_{i j}^{* k l}$,

where $\delta_{i j}$ is the Kronecker delta.

The relationship between the local RVE strain and the average strain (3.22a) is then substituted into the macroscopic level equilibrium equations

$\int_{\Omega} \frac{1}{\left|V_{\mathrm{RVE}}\right|} \int_{V_{\mathrm{RVE}}} C_{i j k l} M_{k l p m} d V_{\mathrm{RVE}} \varepsilon_{i j}^{0}(v) \bar{\varepsilon}_{p m} d \Omega=\int_{\Gamma} t_{i} v_{i} d \Gamma$,

where $\bar{\varepsilon}_{p m}$ and $\varepsilon_{i j}^{0}(v)$ are outside the integral over the RVE since they have constant value over the RVE. Recalling the Eq. (3.7) for the effective stiffness tensor, the final form of the macroscopic equilibrium equation may be written as

$$
\int_{\Omega} \bar{C}_{i j k l} \varepsilon_{i j}^{0}(v) \bar{\varepsilon}_{k l} d \Omega=\int_{\Gamma} t_{i} v_{i} d \Gamma \text {. }
$$

It is important to note that the effective stiffness $\bar{C}_{i j k t}$ calculated by homogenization theory using (3.7) is not dependent on the RVE size due to the periodicity assumption used in determining $M_{i j k l}$. 
This differs from standard mechanics approaches for which the effective stiffness $\bar{C}_{i j k l}$ is dependent on the RVE size due to the St. Venant effect of the applied displacement or traction boundary conditions.

\section{Calculation of effective stiffness: one-dimensional case}

Consider a composite bar composed of two materials that alternate periodically with Young's moduli $E_{1}$ and $E_{2}$ (Fig. 4.1). The basic repeating unit of the microstructure is assumed to be of unit length as is the area of the bar. The general governing equation for homogenization theory (3.20) may be rewritten for the one-dimensional case as:

$\int_{0}^{1} E(x, y) \varepsilon^{*} \varepsilon(v) d y=\int_{0}^{1} E(x, y) \varepsilon(v) d y$

where $E(x, y)$ is the Young's modulus which varies at both the microscopic and macroscopic level, $\varepsilon^{*}$ is the axial fluctuating strain component $(3.13 \mathrm{c})$, and $\varepsilon(v)$ is the axial virtual strain. The following solution is given by Kikuchi (in preparation). First, set $E(x, y)=E$ and rewrite (4.1) as

$\int_{0}^{1} E\left(1-\varepsilon^{*}\right) \varepsilon(v) d y=0$

Applying integration by parts to (4.2) gives

$\int_{0}^{1} v \frac{\partial}{\partial y} E\left(1-\varepsilon^{*}\right) d y+\left.E\left(1-\varepsilon^{*}\right) v\right|_{y=0} ^{y=1}=0$.

The strong form of (4.3) is

$\frac{\partial}{\partial y} E\left(1-\varepsilon^{*}\right)=0$

Integrating (4.4) yields the solution

$E\left(1-\varepsilon^{*}\right)=c(x)$,

where $c(x)$ is constant over the microstructure. To determine $c(x)$, (assuming $E$ is nonzero), (4.5) is integrated over $y$

$\int_{0}^{1}\left(1-\varepsilon^{*}\right) d y=\int_{0}^{1} \frac{c(x)}{E} d y \Rightarrow 1-\left.u^{*}\right|_{0} ^{1}=c(x) \int_{0}^{1} \frac{1}{E} d y$.

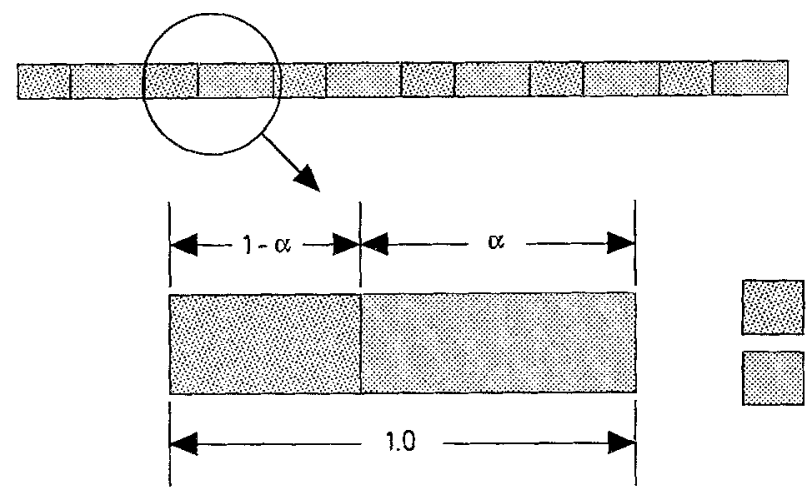

Material 1; Modulus $E_{1}$

Fig. 4.1. Composite bar with alternating materials used for the onedimensional analysis. Total length of the unit cell is 1 . Section 1 of the

Material 2; Modulus $E_{2}$ unit cell has material with modulus $E_{1}$ and length $1-\alpha$. Section 2 of the unit cell has material with modulus $E_{2}$ and length $\alpha$ 
Since the displacements $u^{*}$ must be equal at the cell edges to preserve periodicity, i.e. $u^{*}(1)=u^{*}(0)$, $c(x)$ is

$c(x)=\frac{1}{\int_{0}^{1} \frac{1}{E} d y}$

Simplifying (3.7) for the one-dimensional case (Fig. 4.1) allows the effective stiffness $\bar{E}$ to be written as

$\bar{E}=\int_{0}^{1} E\left(1-\varepsilon^{*}\right) d y=\int_{0}^{1} c(x) d y=c(x)=\frac{1}{\int_{0}^{1-\alpha} \frac{1}{E_{1}} d y+\int_{1-\alpha}^{1} \frac{1}{E_{2}} d y}$.

Evaluating the above integral gives $\vec{E}$ explicitly as:

$\bar{E}=\frac{E_{1} E_{2}}{(1-\alpha) E_{2}+\alpha E_{1}}$.

Next, consider the same composite bar subject to tractions $P$ at each end. The average stress will be equal to $P$ in each phase of the composite. The strain in each section of the composite will be:

$\varepsilon_{1}=\frac{P(1-\alpha)}{E_{1}}, \quad \varepsilon_{2}=\frac{P \alpha}{E_{2}}$.

The effective stiffness of the composite bar is given by elementary mechanics as

$\bar{E}=\frac{\bar{\sigma}}{\bar{\varepsilon}}$.

Since the repeating cell is of unit length and area, the average strain reduces to the sum of the strain in each phase. Combining (4.9) with (4.10) (noting that $\bar{\sigma}=P$ ) gives the effective stiffness using the standard mechanics approach as

$\bar{E}=\frac{E_{1} E_{2}}{(1-\alpha) E_{2}+\alpha E_{1}}$.

Thus, for the one-dimensional case, the effective stiffness computed using homogenization theory and the standard mechanics approach is identical. It is important to note, however, that there is a direct and unique relationship between the applied boundary conditions and the average stress and strain for the one-dimensional standard mechanics approach. Furthermore, there are no Poisson effects in the one-dimensional case.

\section{Computational procedures}

\subsection{Standard mechanics approach}

A displacement based finite element approximation was used to solve (3.3) and (3.4) for $(k, l)=(1,1)$, $(1,2)$, and $(2,2)$ for the two-dimensional test cases. A standard 4-node isoparametric quadrilateral element was used with bilinear shape functions of the form (Kikuchi 1986)

$\hat{N}_{1}(s)=\frac{1}{4}\left(1-s_{1}\right)\left(1-s_{2}\right), \quad \hat{N}_{2}(s)=\frac{1}{4}\left(1+s_{1}\right)\left(1-s_{2}\right)$,

$\hat{N}_{3}(s)=\frac{1}{4}\left(1+s_{1}\right)\left(1+s_{2}\right), \quad \hat{N}_{4}(s)=\frac{1}{4}\left(1-s_{1}\right)\left(1+s_{2}\right)$,

where $s_{i}$ are the coordinates of the "master" element. The displacements $u_{i}$, the virtual displacements $v_{i}$, and the coordinates $x_{i}$ are all approximated by

$u_{i}=\hat{N}_{\alpha}(s) u_{\alpha i}, \quad v_{i}=\hat{N}_{\alpha}(s) u_{\alpha i}, \quad x_{i}=\hat{N}_{\alpha}(s) u_{\alpha i}$. 
Derivatives of quantities with respect to $x_{i}$ are defined by

$\frac{\partial \phi_{i}}{\partial x_{j}}=\frac{\partial N_{\alpha}\left(x_{i}\right)}{\partial x_{j}} \phi_{\alpha i}$

where $N_{\alpha}\left(x_{i}\right)$ is the value of the shape functions in the $x$ coordinate system and $\phi_{\alpha i}$ is the value of a given function at the element nodal points. The shape functions in the physical coordinate system $\boldsymbol{x}$ are then mapped into the coordinates $\boldsymbol{s}$ of the "master" element by

$\frac{\partial N_{\alpha}(\boldsymbol{x})}{\partial x_{i}}=\frac{\partial \hat{N}_{\alpha}(s)}{\partial s_{j}} J_{i j}^{-1}$,

where $J_{i j}^{-1}$ is the inverse of the Jacobian. Discretizing the left-hand side of (3.3) and (3.4) using the procedure described above gives the element stiffness matrix

$K_{i \alpha j \beta}^{e}=\sum_{i x=1}^{2} \sum_{i y=1}^{2} C_{i j k l} \frac{\partial \hat{N}_{\alpha}}{\partial s_{m}} J_{j m}^{-1} \frac{\partial \hat{N}_{\beta}}{\partial s_{n}} J_{l n}^{-1}|J| W_{i x} W_{i y}$,

where $|J|$ is the determinant of the Jacobian, $W_{i x}$ and $W_{i y}$ are the Gauss weighting points, and $K_{i \alpha j \beta}^{e}$ represents the element stiffness matrix which is stored in an array at $(2 \alpha-2+i, 2 \beta-2+j)$ for $\alpha, \beta=1,2,3,4$ and $i, j=1,2$. In all cases, a $2 \times 2$ Gauss integration scheme was used to integrate the element stiffness matrix. The element stiffness matrices were assembled in the usual manner to the global stiffness matrix.

For the applied displacement approach, the specified displacements were imposed by modifying both the global stiffness matrix and the global load vector with a penalty parameter at the appropriate degrees of freedom (Kikuchi 1986). This parameter was generally chosen to be $\left(1.0 \times 10^{7}\right) C_{1111}$. The final discretized form of the equilibrium equations was

$K_{i j} u_{j}^{k l}=f_{j}^{k l}$.

For the applied traction approach, the final form of the discretized equilibrium equations is the same as (5.2). However, to avoid a singular stiffness matrix resulting from the absence of constrained displacements, a regularization method was applied (Kikuchi 1986)

$K_{i \alpha j \beta}^{e}=\sum_{i x=1}^{2} \sum_{i y=1}^{2}\left(C_{i j k l} \frac{\partial \hat{N}_{\alpha}}{\partial s_{m}} J_{j m}^{-1} \frac{\partial \hat{N}_{\beta}}{\partial s_{n}} J_{l n}^{-1}+\varepsilon \delta_{i k} \hat{N}_{\alpha} \hat{N}_{\beta}\right)|J| W_{i x} W_{i y}$,

where $\varepsilon$ is the regularity parameter (chosen to be $\varepsilon=10^{-5} C_{1111}$ ), $\delta_{i k}$ is the Kronecker delta, and the other terms are as defined previously. The right-hand side of (3.3) is discretized using two node line elements along the boundary where the traction is applied.

Once the discretized versions of (3.3) or (3.4) are solved, the strain $\varepsilon_{p m}^{k l}$ is calculated at the element centroid. The average strain $\bar{\varepsilon}_{p m}^{k l}$ is obtained by integrating the displacements over the RVE boundary (3.1). Equation 3.1 is used to calculate the average strain because the relationship between $\bar{\varepsilon}_{p m}^{k l}$ and $\varepsilon_{i j}^{k l}$ is not defined in the void. However, the relationship can be approximated by assuming the void is replaced by a soft material whose stiffness in the limit goes to zero (Suquet 1987). This makes the application of (3.1) valid.

The local structure tensor $M_{i j p m}$ can be calculated at the element centroid once $\bar{\varepsilon}_{p m}^{k l}$ and $\varepsilon_{i j}^{k l}$ are known. Taking advantage of the symmetries in $\bar{\varepsilon}_{p m}^{k l}, \varepsilon_{i j}^{k l}$, and $M_{i j p m}$, it is possible to calculate $M_{i j p m}$ by solving three sets of matrix equations (for 2D)

$\left\{\begin{array}{c}\varepsilon_{i j}^{11} \\ \varepsilon_{i j}^{22} \\ \varepsilon_{i j}^{12}\end{array}\right\}=\left[\begin{array}{lll}\varepsilon_{11}^{11} & \varepsilon_{22}^{11} & 2 \varepsilon_{12}^{11} \\ \varepsilon_{11}^{22} & \varepsilon_{22}^{22} & 2 \varepsilon_{12}^{22} \\ \varepsilon_{11}^{12} & \varepsilon_{22}^{12} & 2 \varepsilon_{12}^{12}\end{array}\right]\left\{\begin{array}{l}M_{i j 11} \\ M_{i j 22} \\ M_{i j 12}\end{array}\right\}$.

Once $M_{i j k l}$ is known, the effective stiffness $\bar{C}_{i j k l}$ is calculated using (3.7) by integrating over the discretized RVE and dividing by the total volume of the RVE (including the volume of the void if present). Since only one value of $M_{i j k l}$ and $C_{i j k l}$ are given for each element, the integral in (3.7) 


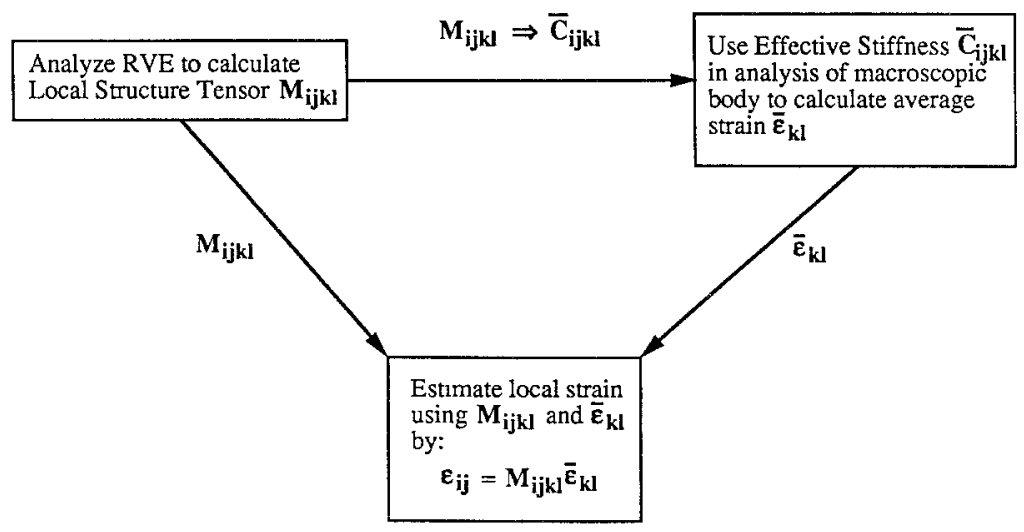

Fig. 5.1. Computational procedure for RVE approach. Schematic outline of the computational procedure used in the RVE approach to composite material analysis. First, the RVE is analyzed using the chosen approach (homogenization or standard mechanics) to calculate the local structure tensor $M_{i j k l}$ and subsequently the effective stiffness tensor $\vec{C}_{i j k l}$. The average strain $\bar{\varepsilon}_{k l}$ is then calculated in a macroscopic analysis assuming the composite structure to be a locally homogeneous material with stiffness $\bar{C}_{i j k l}$. Finally, the local strain $\varepsilon_{\imath}$ in each RVE is estimated from the average strain and the local structure tensor

is approximated by multiplying the integrand in (3.7) in each element by the element's volume and summing over all elements.

After the effective stiffness $\bar{C}_{i j k l}$ has been determined, Eq. 3.24 can then be solved to determine the average strain $\bar{\varepsilon}_{k l}$ resulting in a homogeneous body under given boundary conditions. The strain at any microstructural location within the global body is then calculated by post-processing the average strain $\bar{\varepsilon}_{k l}$ with the local structure tensor $M_{i j k l}$. A flowchart of the computational procedure is given in Fig. 5.1.

\subsection{Homogenization approach}

A displacement based finite element approach was also used to solve the general case of the homogenization microscopic equilibrium (3.20). Discretizing the left-hand side of (3.20) gives the same form of element stiffness matrix as for the applied displacement approach (5.1)

$K_{i \alpha j \beta}^{e}=\sum_{i x=1}^{2} \sum_{i y=1}^{2} C_{i j k l} \frac{\partial \hat{N}_{\alpha}}{\partial s_{m}} J_{j m}^{-1} \frac{\partial \hat{N}_{\beta}}{\partial s_{n}} J_{l n}^{-1}|J| W_{i x} W_{i y}$

which is stored in a two-dimensional array at $(2 \alpha-2+i, 2 \beta-2+j)$ for $\alpha, \beta=1,2,3,4$ and $i, j=1,2$. A $2 \times 2$ Gauss integration scheme is again used to evaluate both the element stiffness matrix and element load vector. Discretizing the right-hand side of $(2.20)$ using the procedure outlined in Sect. 4.1 gives the following element load vector

$f_{i \alpha}^{(e) k l}=\sum_{i x=1}^{2} \sum_{i y=1}^{2} \sigma_{i j}^{* k l} \frac{\partial \hat{N}_{\alpha}}{\partial s_{m}} J_{j m}^{-1}|J| W_{i x} W_{i y}$

where $\sigma_{i j}^{* k l}$ is defined in (3.19) and the other terms are as defined previously. The element load vector $f_{i \alpha}^{(e) k l}$ is stored in a one-dimensional array at $(2 \alpha-2+i)$, for $\alpha=1-4$ and $i=1-2$.

After assembling the element stiffness matrix and the element load vector, the displacements at opposite sides of the RVE are constrained to be equal using a penalty method to ensure that $\varepsilon_{i j}^{* k l}$ is periodic in (3.20). The implementation of this displacement constraint differs slightly from the constraints used in the applied displacement approach. Let $i$ and $j$ be the degrees of freedom on opposite sides of the RVE that are constrained to displace equally. Then the global stiffness matrix $K_{i j}$ is modified as follows

$K_{i i}=K_{i i}+\lambda, \quad K_{j j}=K_{j j}+\lambda, \quad K_{i j}=K_{i j}-\lambda$, 
where $K_{i j}$ is the $i j^{\text {th }}$ entry of the global stiffness matrix and $\lambda$ is the penalty parameter, chosen to be $\left(1.0 \times 10^{7}\right) C_{1111}$. Finally, because the present formulation does not include boundary effects, the local structure tensor $M_{i j k l}$ is defined up to an additive constant using the purely periodic constraints (Guedes 1990). To constrain the global stiffness matrix against rigid body modes, one node of the finite element model is constrained not to displace using the same penalty approach as in the applied displacement approach. The choice of node does not influence the final value of the effective elastic constants $\bar{C}_{i j k l}$ (Guedes 1990).

After the discretized version of (3.20) is solved, the fluctuating strain components $\varepsilon_{i j}^{* k l}$ are determined at the element centroids. These fluctuating strain components are then used to calculate the local structure tensor $M_{i j k l}$ using (3.22b). Note that $M_{i j k l}$ is calculated by superposition with the identity tensor unlike the standard approaches in which $M_{i j k l}$ is back calculated by solving a system of linear equations. This difference is much more noticeable for $3 \mathrm{D}$ problems in which six sets of $6 \times 6$ matrix equations need to be solved for standard approaches while $(3.22 \mathrm{~b})$ may be used in the homogenization approach to determine $M_{i j k l}$ without any more difficulty.

After $M_{i j k l}$ is determined, the effective stiffness tensor is calculated using (3.7) and the same method as discussed with standard approaches. The procedure outlined in Fig. 5.1 is followed to calculate first the average strain $\bar{\varepsilon}_{k l}$ in the global body and then the local strain $\varepsilon_{k l}$ at each point in the global body.

\section{Two-dimensional comparison: methods}

Two-dimensional cellular structures with solid volume fractions of 30\%,50\%,70\%, and $90 \%$ were analyzed. For the standard mechanics approach RVE sizes of 1,4,9,16, and 25 cells (here "cell" refers to the basic repeating unit of the periodic material) were analyzed (Fig. 6.1) for each solid volume fraction. Both uniform traction and uniform displacement boundary conditions were applied for the standard mechanics approach. For the homogenization analysis only one cell was analyzed for each volume fraction since the periodicity assumption gives the same result for any number of cells. The solid modeling and mesh generation program PATRAN (PDA Engineering, Costa Mesta, CA) was used to generate the finite element mesh for each case.
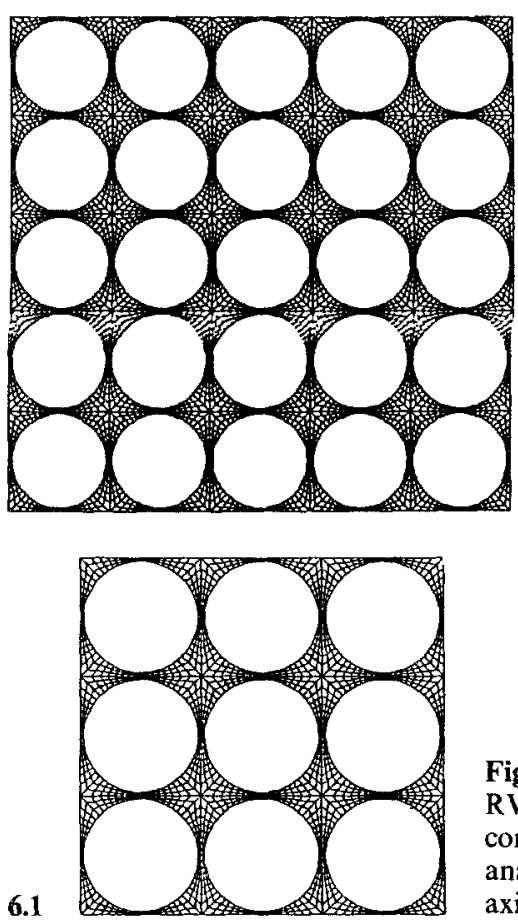

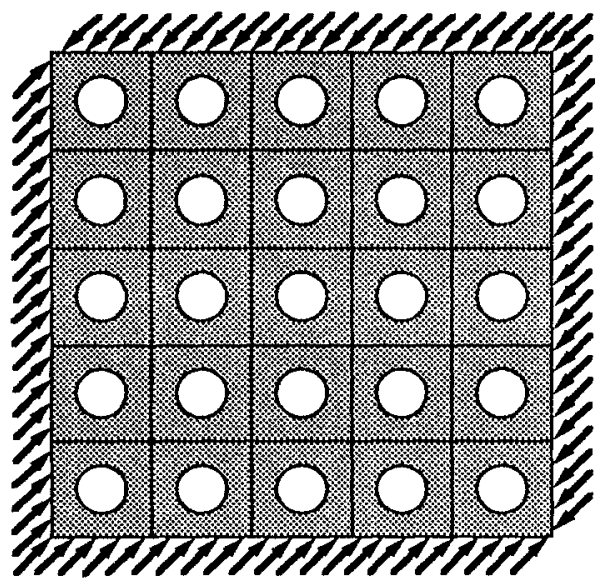

6.2

Figs. 6.1 and 6.2. 6.1 Sample finite element meshes used for RVE analysis. A 25-celled RVE is shown on top and a 9-celled is shown on the bottom. 6.2 Off-axis boundary conditions used for comparison of local strain energy density from direct and RVE analyses. The applied displacements and tractions are oriented at $45^{\circ}$ to the principal axis of the material. 


\subsection{Effective stiffness comparisons}

The effective stiffness tensor $\bar{C}_{i j k l}^{\prime}$ as a function of orientation $\theta$ was calculated for each case using the standard transformation

$\bar{C}_{i j k l}^{\prime}=\bar{C}_{m n p q} a_{i m} a_{j n} a_{k p} a_{l q}$,

where $\bar{C}_{m n p q}$ is the effective stiffness tensor in the principal orientation of the microstructure and $a_{i m}$ is the direction cosine tensor for a given orientation. The percent difference between $\bar{C}_{i j k l}^{\prime}$ determined using the standard mechanics approach and $\bar{C}_{i j k l}^{\prime}$ determined using homogenization theory was then calculated for $\theta$ between $0^{\circ}$ and $90^{\circ}$.

\subsection{Comparison of local strain energy density distributions}

The local strain energy density $(U)$ distribution predicted by each approach was compared to direct estimates $U^{D}$ from a complete finite element analysis for porous cell structures of $30 \%, 50 \%$, $70 \%$, and $90 \%$ solid volume fraction. Each structure contained 25 cells and was subject to two different boundary conditions: 1) an off axis boundary displacement and 2) an off axis boundary traction (Fig. 6.2). One celled RVEs of $30 \%, 50 \%, 70 \%$, and $90 \%$ volume fraction were analyzed using each RVE approach (homogenization, uniform displacement, and uniform traction) to determine $\bar{C}_{i j k l}$ and the local structure tensor $M_{i j k l}$. Homogeneous materials with stiffness $\bar{C}_{i j k l}$ of the same size as the original cell structures were analyzed under the two different boundary conditions to determine $\bar{\varepsilon}_{i j}$. The average stress and strain distributions were then post-processed using (3.6) to determine $\varepsilon_{i j}$ and $\sigma_{i j}$ from which $U$ was calculated. The percent difference between the direct estimate and each microstructural analysis estimate (calculated as $\left.100 \frac{U^{D}-U}{U^{D}}\right)$ was
then plotted on the original structure.

\section{Two-dimensional comparison: results}

\subsection{Effective stiffness comparisons}

The largest difference between $\bar{C}_{i j k l}^{\prime}$ calculated using applied displacements and $\bar{C}_{i j k l}^{\prime}$ calculated using homogenization theory occurred for the $30 \%$ solid volume fraction material. Both $\bar{C}_{111}^{\prime}$ and $\bar{C}_{1122}^{\prime}$ converged from above to the homogenization predictions as the number of cells in the RVE increased. The largest difference for $\bar{C}_{1111}^{\prime}$ was $+72 \%$ and occurred at a $45^{\circ}$ orientation while the largest difference for $\bar{C}_{1122}^{\prime}$ was $+1000 \%$ and occurred at a $0^{\circ}$ orientation. The applied displacement prediction of the shear modulus, $\bar{C}_{1212}^{\prime}$, converged from below to the homogenization prediction as a function of orientation. The largest difference of $-54 \%$ for $\bar{C}_{1212}^{\prime}$ occurred at a $45^{\circ}$ orientation. The maximum difference between the homogenization and applied displacement predictions of $\bar{C}_{1111}^{\prime}, \bar{C}_{1122}^{\prime}$, and $\bar{C}_{1212}^{\prime}$ for the $30 \%$ volume fraction materials are plotted against the inverse of the number of cells in the RVE used in the applied displacement analysis in Fig. 7.1. The applied displacement predictions converged to the homogenization predictions as approximately linear functions of the number of cells in the RVE. The differences between $\bar{C}_{1111}^{\prime}, \bar{C}_{1122}^{\prime}$, and $\bar{C}_{1212}^{\prime}$ for the $90 \%$ volume fraction material as a function of RVE size (Fig. 7.2) show nearly linear convergence, with much smaller differences between the stiffness predictions of the two approaches. In general, increasing the RVE size or the solid volume fraction decreased the difference between the applied displacement and homogenization estimates of effective stiffness.

The differences between $\bar{C}_{i j k l}^{\prime}$ calculated using applied traction boundary conditions and $\bar{C}_{i j k l}^{\prime}$ calculated using homogenization theory were generally larger than differences found using applied displacements. The applied traction predictions of $\bar{C}_{111 \mathrm{i}}^{\prime}$ converged from below while the predictions of $\bar{C}_{1122}^{\prime}$ converged from above to the homogenization predictions. The largest difference 


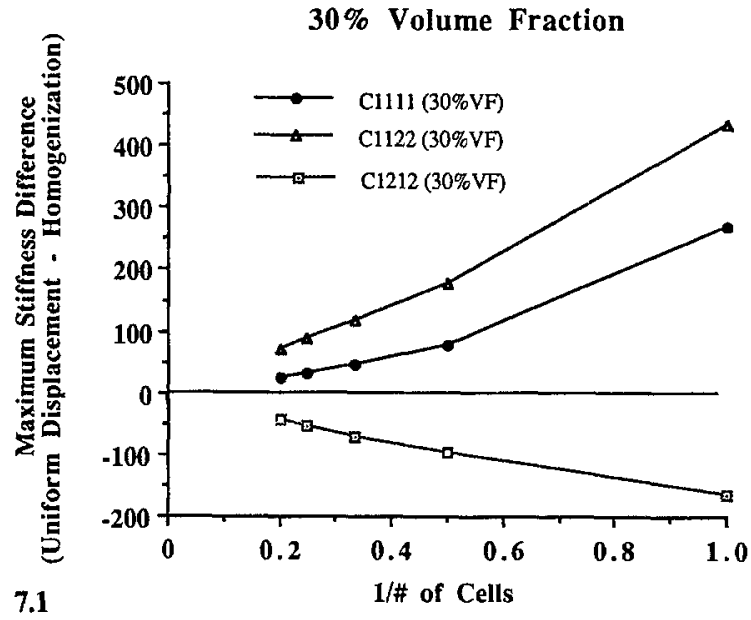

$30 \%$ Volume Fraction

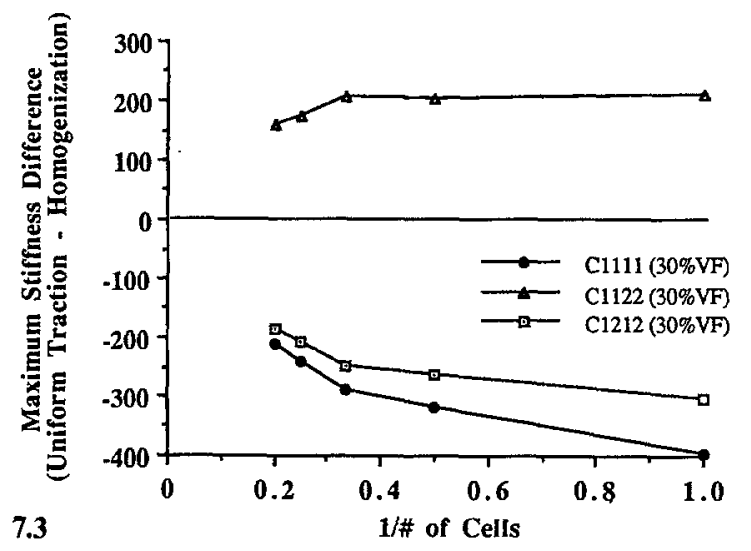

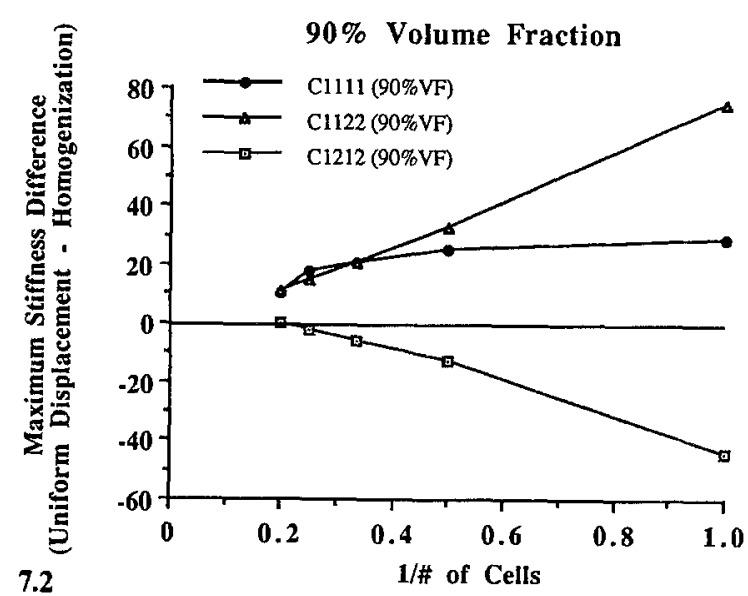

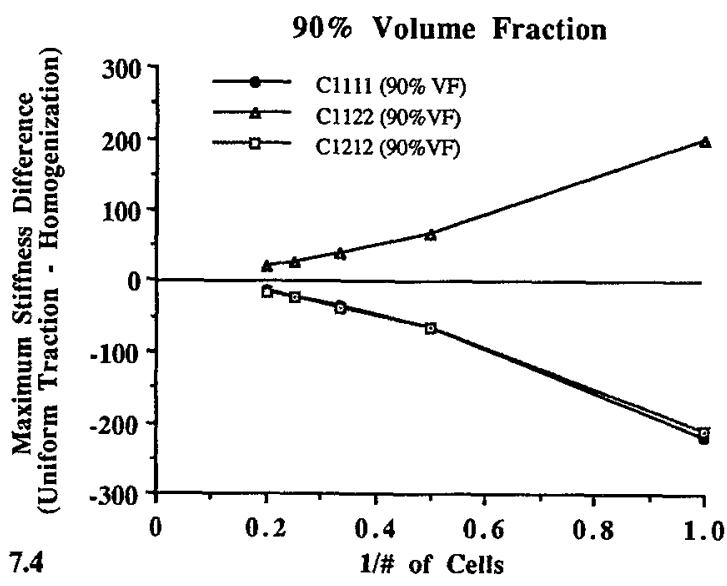

Figs. 7.1-7.4. 7.1 Maximum difference between applied displacement and homogenization predictions of effective stiffness terms $\bar{C}_{1111}, \bar{C}_{1122}, \bar{C}_{1211}$ of the $30 \%$ volume fraction material as a function of the inverse number of cells along one direction of the RVE. For one-cell RVE analyses, there are large differences between the applied displacement approach and the homogenization analysis. As the number of cells analyzed using applied displacements is increased to $5 \times 5$, the stiffness predictions converge to that of the 1-cell homogenization analysis. 7.2 Maximum difference between applied displacement and homogenization predictions of effective stiffness terms $\bar{C}_{111}, \bar{C}_{1122}, \bar{C}_{1212}$ of the $90 \%$ volume fraction material as a function of the inverse number of cells along one direction of the RVE. Although the initial stiffness predictions differences are much less than the $30 \%$ material, the same convergence pattern is seen. 7.3 Maximum difference between applied traction and homogenization predictions of effective stiffness terms $\bar{C}_{1111}, \bar{C}_{1122}, \bar{C}_{1212}$ of the $30 \%$ volume fraction material as a function of the inverse number of cells along one direction of the RVE. The differences in stiffness prediction are much larger than the differences found for the applied displacement approach and the convergence is much slower. 7.4 Maximum difference between applied traction and homogenization predictions of effective stiffness terms $\bar{C}_{1111}, \bar{C}_{1122}, \bar{C}_{1212}$ of the $90 \%$ volume fraction material as a function of the inverse number of cells along one direction of the RVE. For this case, the differences in the 1-celled RVE analysis are similar to the applied displacement case. In addition, the convergence for this case is much faster than for the $30 \%$ volume fraction material analyzed using the applied traction approach

for $\bar{C}_{1111}^{\prime}(-60 \%)$ and $\bar{C}_{1122}^{\prime}(+500 \%)$ both occurred at a $0^{\circ}$ orientation for the $30 \%$ volume fraction material. The applied traction predictions of $\bar{C}_{1212}^{\prime}$ converged from below to the homogenization predictions, with the largest difference of $-100 \%$ occurring at a $45^{\circ}$ orientation for the $30 \%$ volume fraction material. Plots of the maximum difference between the homogenization predictions and the applied traction predictions of $\bar{C}_{1111}^{\prime}, \bar{C}_{1122}^{\prime}$, and $\bar{C}_{1212}^{\prime}$ for the $30 \%$ volume fraction material (Fig. 7.3) show that the applied traction predictions converged to the homogenization predictions as approximately a linear function of RVE size. Similar plots for the $90 \%$ volume fraction material (Fig. 7.4), also show approximately linear convergence between the applied traction and homogenization predictions as a function of RVE size. As with the applied 
displacement approach, increasing the RVE size or the solid volume fraction decreased the difference between the applied traction and homogenization estimates of the effective stiffness.

\subsection{Comparison of local strain energy density distributions}

Homogenization estimates of the local strain energy density $U$ showed two different trends, depending on the applied boundary condition. The homogenization estimates were very close to the direct estimates for the off axis traction boundary conditions (Fig. 7.5 shows the $30 \%$ volume fraction case). For all volume fractions the homogenization estimates were within $\pm 30 \%$ of the direct estimates, except in small regions close to the boundary. The errors near the boundary increased slightly as the volume fraction decreased, but errors greater than $\pm 30 \%$ were only found within approximately one half cell length of the boundary. Agreement between homogenization and direct estimates under off axis boundary displacement conditions were fairly close for the $30 \%$ (Fig. 7.6) volume fraction case, but became progressively worse as the solid volume fraction increased. With increasing solid volume fraction, the off axis displacement case errors progressed inward from the lower left and upper right side boundaries parallel to the direction of the applied displacements.

Local strain energy density estimates from the applied displacement approach showed similar trends for both the off-axis traction and displacement boundary conditions. For the off-axis traction case, errors increased in magnitude from generally less than $\pm 30 \%$ at $90 \%$ volume fraction to over $+70 \%$ to $+90 \%$ at $30 \%$ volume fraction (Fig. 7.7). The errors at all volume fractions were distributed in periodic patterns over the porous structures and increased consistently within these periodic patterns as the volume fraction decreased. The errors for the off-axis displacement case increased from between $\pm 30 \%$ and $+60 \%$ at $90 \%$ volume fraction to over $-70 \%$ to $-90 \%$ for the entire $30 \%$ volume fraction material (Fig. 7.8). Errors in the applied displacement predictions and the homogenization estimates were similar for the $90 \%$ volume fraction material under displacement boundary conditions. With decreasing volume fraction, however, the errors were increasingly distributed in periodic patterns in the same manner as the off-axis traction case.

The local strain energy estimates from the applied traction approach, like those from the applied displacement approach, showed similar error trends for both off-axis boundary conditions.

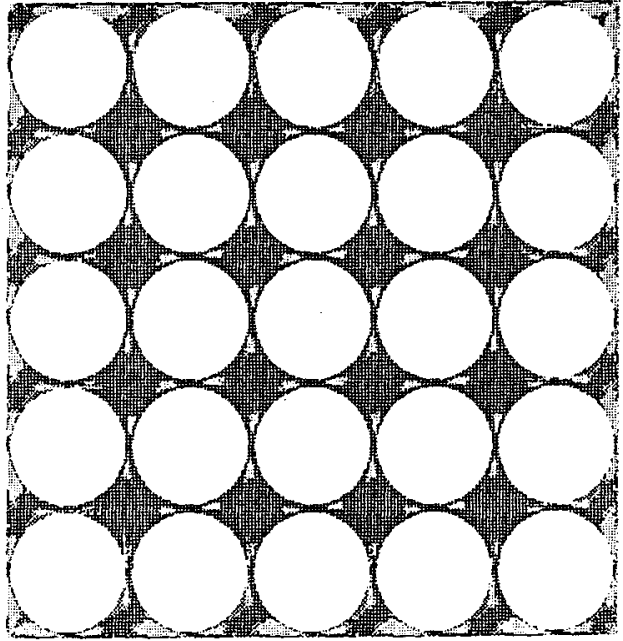

7.5

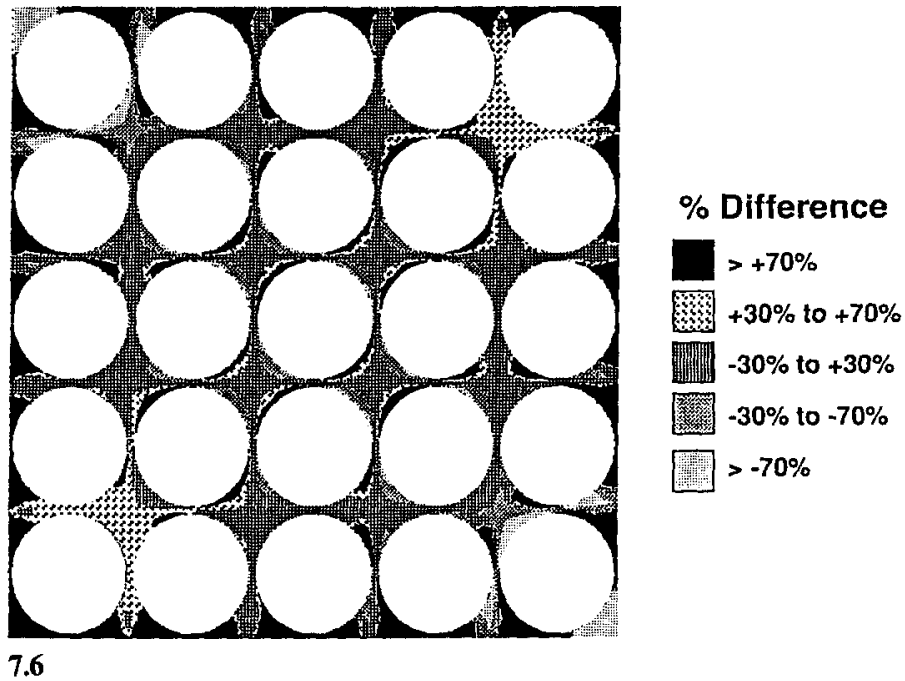

7.6

Figs. 7.5 and 7.6. Percent difference in strain energy density calculated by direct finite element analysis and by the homogenization RVE approach 7.5 for the 25-celled structure under off-axis boundary tractions. The homogenization estimates are with $\pm 30 \%$ over the entire structure except for small regions near the boundary; 7.6 for the 25-celled structure under off-axis boundary displacements. For this case, the homogenization estimates are within $\pm 30 \%$ of the direct estimate over the interior of the structure approximately one half cell length away from the boundary. Close to the boundary, the errors become much larger, due to boundary layer effects 


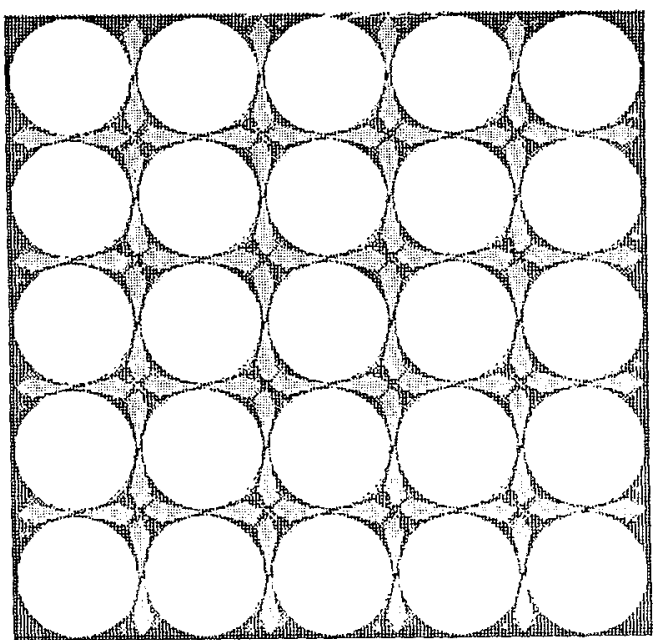

7.7

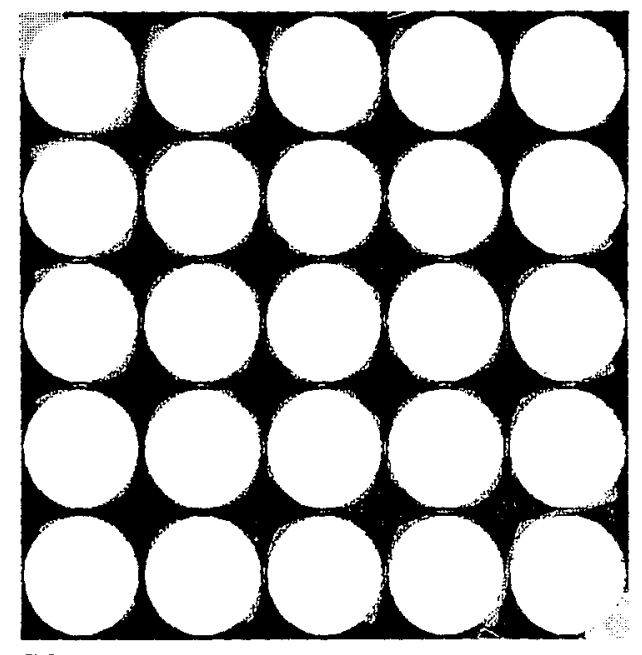

7.8
$\%$ Difference

$>+70 \%$

$+30 \%$ to $+70 \%$

鼠留 $-30 \%$ to $+30 \%$

$-30 \%$ to $-70 \%$

[7] $>-70 \%$

Figs. 7.7 and 7.8. Percent difference in strain energy density calculated by direct finite element analysis and by the applied displacement RVE approach 7.7 for the 25-celled structure under off-axis boundary tractions. (The errors for this case are much larger homogenization analysis and are distributed periodically over the whole structure. The errors range from $+30 \%$ to over $-70 \%$ ); 7.8 for the 25 -celled structure under off-axis boundary displacements. (The errors for this case are again much larger than the homogenization analysis and are distributed periodically over the whole structure. The errors in this case are almost all over $+70 \%$ )

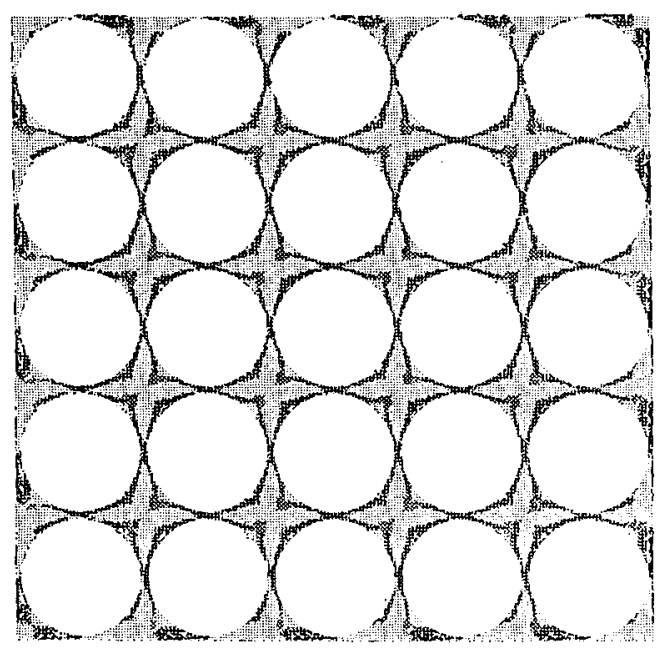

7.9

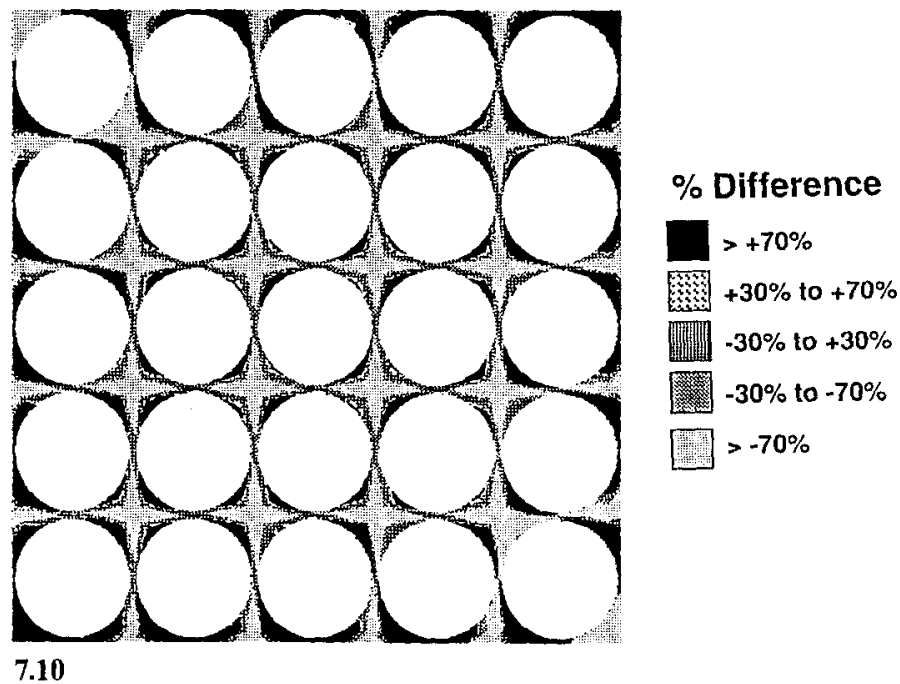

Figs. 7.9 and 7.10. Percent difference in strain energy density calculated by direct finite element analysis and by the applied traction RVE approach $\mathbf{7 . 9}$ for the 25-celled structure under off-axis boundary displacements. (The errors for this case are also much larger than the homogenization analysis and are also distributed periodically over the whole structure. The errors range from $+30 \%$ to over $-70 \%$ ); 7.10 for the 25 -celled structure under off-axis boundary tractions. (The errors are distributed periodically over the entire structure and fluctuate rapidly between $-70 \%$ to $+70 \%$ )

Errors for the off-axis traction boundary condition increased from between $\pm 30 \%$ and $+60 \%$ at $90 \%$ volume fraction to over $+70 \%$ to $+90 \%$ at $30 \%$ volume fraction (Fig. 7.9 ). The errors at all volume fractions were distributed in periodic patterns similar to those of the applied displacement approach. The errors consistently increased within these periodic patterns as the solid volume fraction decreased. Errors for the off-axis displacement boundary condition were somewhat similar to the homogenization approach at $90 \%$ volume fraction (between $\pm 30 \%$ to over $+60 \%$ ), but increased significantly at $30 \%$ solid volume fraction (Fig. 7.10 ) to over $-70 \%$ to $-90 \%$. These errors were increasingly arranged in periodic patterns as the solid volume fraction decreased. 


\section{Analysis of a composite plate}

\subsection{Methods}

A plate consisting of a solid homogeneous material bonded to a two-layer cellular porous material with a solid volume fraction of $30 \%$ (Fig. 8.1) was used to examine the accuracy of the RVE approaches for large boundary layer effects and large $\eta$. The plate was fixed at the left end and subject to uniform compressive displacement of 0.001 at the other end. The homogeneous material and the material in the solid part of the void structure had the same elastic properties. The complete structure was discretized using 96004 -node bilinear quad elements to directly estimate the strain energy density.

The plate was also analyzed using the applied traction, applied displacement, and homogenization RVE approaches using the computational procedure outlined in Fig. 5.1. First, a 1-celled RVE model of the void section was analyzed using each microstructural analysis method. (Note: For the homogeneous section, no microstructural analysis was done since the local structure tensor reduces to the identity tensor and the average strain is equal to the local strain). Next, a homogeneous material of the same dimensions as the plate with the effective stiffness from the RVE approaches was analyzed using the same boundary conditions as the direct analysis to calculate the average strain. Then, for each RVE approach, the local strain distribution was estimated using (3.6) after which the local strain energy density was calculated. For each RVE approach, the difference between the RVE estimate and the direct estimate was calculated as a percentage of the direct estimate and plotted on the finite element mesh of the complete plate.

\subsection{Results}

The results for the homogenization analysis (Fig. 8.2a) show that estimates within the porous layer are within $\pm 15 \%$ of the direct estimates for areas one half cell length away from the free boundary. Near to the boundary with the homogeneous material layer, the errors increased to between $+30 \%$ and $+45 \%$ over the direct estimates. The largest errors of over $\pm 100 \%$ occurred at the free boundary. In the homogeneous material layer, the homogenization estimates were within $\pm 5 \%$, except at the boundary with porous material where the errors increased slightly to $+15 \%$.

The results of the standard approaches were not as accurate as those of the homogenization analyses. The applied displacement approach underestimated the direct estimates of strain energy density by at least $100 \%$ over the majority of the porous section (Fig. $8.2 \mathrm{~b}$ ). Near the boundary between the two materials the errors were also larger than $-100 \%$. In the homogeneous section, the errors were similar to the homogenization analysis. As with the analysis of the 25 celled porous materials, the errors produced by the applied displacement approach were distributed periodically over the porous section of the plate.

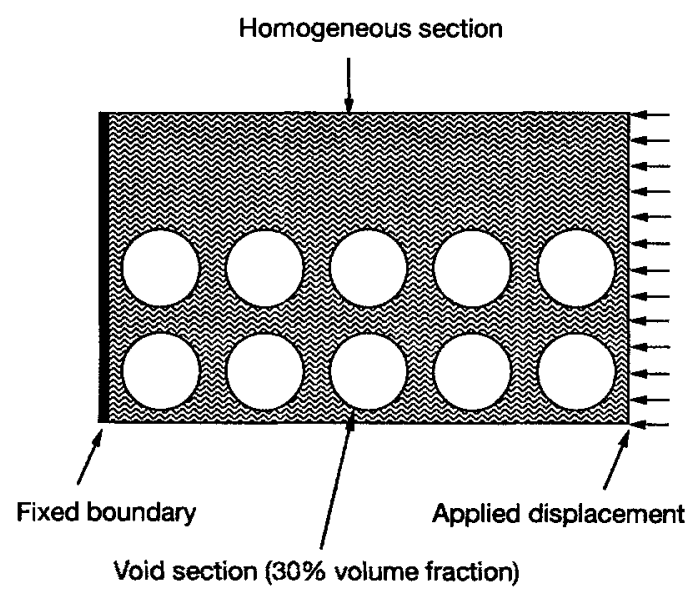

Fig. 8.1. Composite plate structure consisting of a homogeneous material bounded to a porous material with $30 \%$ volume fraction. The structure is fixed at one end and subject to a uniform compressive displacement at the other end. This was used to test the effects of large $\eta$ ( 0.5 through the thickness of the plate) and boundary layer effects at the interface between the two materials and at the free boundary for each RVE analysis approach 


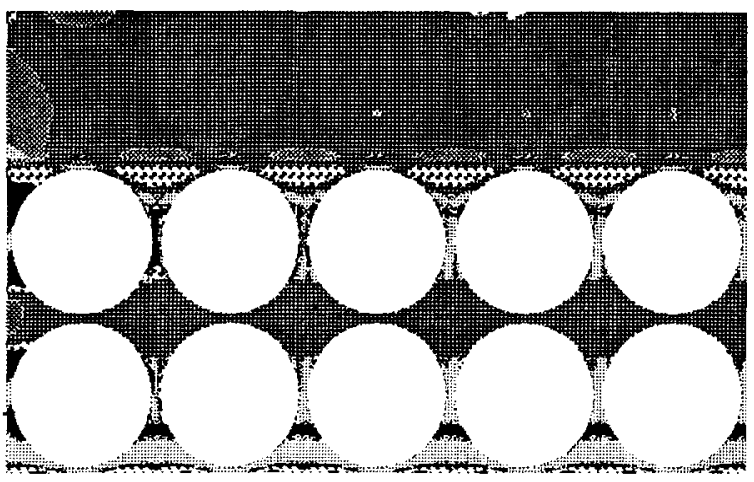

a

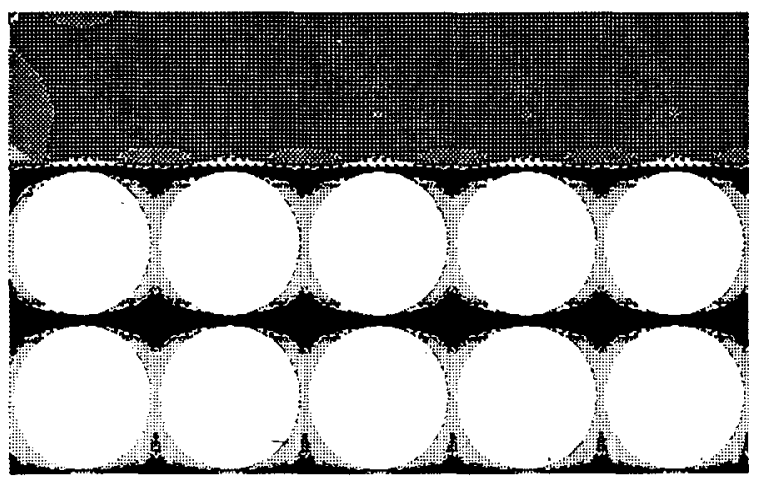

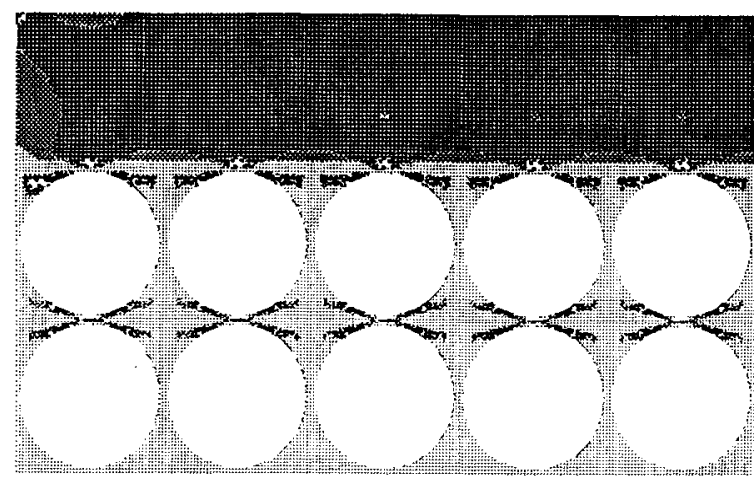

b

\section{$\%$ Difference}

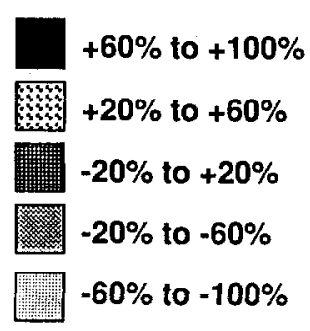

$\mathbf{C}$

Fig. 8.2 a-c. Percent difference between strain energy density calculated by direct finite element analysis and homogenization (a), between direct analysis and the applied displacement approach (b), and between direct analysis and the applied traction approach (c). The homogenization analysis gives estimates within $\pm 20 \%$ of the direct analysis over large sections of the porous structure, except near the free boundary. The other two standard mechanics approaches give much poorer estimates of the strain energy density with errors above $-60 \%$ to $-100 \%$ for the applied displacement approach and errors ranging between $+100 \%$ and $-100 \%$ for the applied traction approach.

The applied traction approach either overestimated or underestimated the direct estimates of strain energy density by nearly $100 \%$ (Fig. 8.2c). As with the applied displacement approach, the errors were distributed periodically over the porous structure. Errors in the porous section at the boundary between the materials were generally between $+85 \%$ and $+100 \%$. Errors in the homogencous matcrial layer were similar to both the homogenization and applied displacement approaches.

\section{Discussion}

The purpose of this paper was to compare homogenization and standard mechanics estimates of effective stiffness and local strain energy distributions for porous materials with periodic microstructures. The results indicated that the two methods are equivalent for a restricted class of one-dimensional periodic composites. However, for two- and three-dimensional periodic porous composites homogenization theory gave much more accurate estimates of local strain energy than standard mechanics materials compared to direct analyses of the materials. Furthermore, the effective stiffness calculated using standard mechanics approaches converged towards the homogenization effective stiffness as $\eta \rightarrow 0$. Given that estimates of effective stiffness are more accurate as $\eta \rightarrow 0$, this results shows that homogenization theory gives more accurate estimates of effective stiffness for periodic materials than standard mechanics approaches with less computational effort. 
For the one-dimensional case, the different formulations of the standard mechanics approaches and homogenization theory gave the same estimate of effective stiffness. In the one-dimensional case, however, there is a one-to-one relationship between the applied traction and the average stress in each phase of the composite bar. Also, the average strain and the local strain are the same in each phase. Since the fluctuation of the local RVE strain about the average RVE strain is zero and the average strain is uniquely determined by the applied traction, the homogenization and standard mechanics approaches are equivalent for the one-dimensional case.

The two- and three-dimensional cases differ in two ways from the one-dimensional case. First, the local RVE strain in the 2D and 3D cases is not equal to the average RVE strain but fluctuates about the average RVE strain. Second, the relationship between the average RVE strain and any applied boundary condition is not unique (see Eq. 3.1 and 3.2). The first consideration is relevant for calculating the local strain distribution from the average strain distribution. Since homogenization theory and standard mechanics approaches make different assumptions about the relationship between the local and average RVE strain, they will produce different predictions of the local RVE strain for the 2D and 3D cases. The second consideration is relevant for calculation of the effective stiffness. The homogenization predictions are determined solely by the assumption of a periodic microstructure. The standard mechanics approaches assume a priori boundary conditions which fix the average RVE strain. Therefore, the effective stiffness is determined to some degree by the choice of boundary conditions.

Both the applied displacement and applied traction predictions of $\bar{C}_{\imath j k l}$ converged to the homogenization prediction for all volume fractions as the RVE size increased. The difference between homogenization and standard mechanics estimates of $\bar{C}_{i j k l}$ also converged as the solid volume fraction increased. Increasing the RVE size or increasing the solid volume fraction both reduce the effect of boundary conditions on the variation of the RVE local strain distribution for the standard mechanics approaches. Differences between homogenization and standard mechanics predictions of $\bar{C}_{i j k l}$ are strongly related to the void/RVE size ratio, being reduced as the void/RVE size ratio is reduced. As the void to RVE size is reduced, fluctuations of the local strain about a mean strain are reduced, and in the limit the local strain fluctuations about the mean reach a fixed value (Hill 1963). The homogenization predictions are unaffected by RVE size since the strain distribution is periodic and increasing RVE size does not change the local strain fluctuation about a mean value.

The local strain energy predicted by homogenization analysis was within $30 \%$ of that computed directly for most cases. The largest errors occurred at the traction and free boundaries for both the two-dimensional porous composites and the composite plate. The location of errors is consistent with the discussions of Lions (1981), Bakhvalov and Panasenko (1989), Dumontet (1985), and Sanchez-Palencia (1987). They all note that the homogenization formulation as applied here does not account for large fluctuations in stress and strain which occur close to the boundary. The boundary layer effects for the cases studied, however, decayed rapidly and seem to diminish within half the length of one unit cell away from the boundary. For locally periodic materials, the largest errors will most likely occur at the boundary between dissimilar materials. The results of this study suggest that homogenization theory will give accurate estimates (within $30 \%$ of a direct analysis for most cases) of local stress for locally periodic composites except in a thin layer at the boundary between dissimilar materials. Furthermore, it is possible to improve the accuracy of homogenization analyses near boundaries by including boundary layer terms as discussed by Bakhvalov and Panasenko (1989) and Dumontet (1985).

The local strain energy predicted by the standard mechanics analyses differed by more than $70 \%$ from direct analyses for all cases studied. The distribution of error was strikingly different for the standard mechanics approach compared to the homogenization approach. Errors in the standard mechanics predictions were distributed periodically over the entire area of the composite. The periodic distribution of error strongly suggests that the local stress and strain fields predicted by the standard mechanics analyses did not agree with the dominant periodic distribution of stress and strain within the interior of the composite. It appears that standard mechanics analyses utilizing applied tractions or displacements will always engender large local stress and strain errors in materials with periodic microstructure. Furthermore, unlike homogenization theory, standard 
mechanics approaches are not based on a sound mathematical foundation in which the accuracy of the approach is assessed a priori for a given $\eta$. For periodic materials with finite $\eta$, homogenization theory provides clearly superior results compared to standard mechanics approaches.

\section{Conclusions}

There were two major findings from this paper concerning the linear elastic analysis of periodic porous composites using standard mechanics and homogenization RVE-based methods. First, the prediction of effective stiffness by standard mechanics approaches using representative volume elements with increasing number of unit cells converged to the homogenization prediction obtained with an RVE consisting of one unit cell. Second, homogenization predictions of local strain energy density were generally within $30 \%$ of direct analyses of periodic porous composites with $\eta=0.2$ while standard mechanics predictions differed from direct analyses by more than $70 \%$. These two results clearly indicate that homogenization theory is preferable over standard mechanics approaches for analyzing locally periodic composites like wood and human bone tissue with finite $\eta$ as well as globally periodic composites. Furthermore, the ability to modify the analysis through the use of boundary layer terms or additional terms in the asymptotic expansion (Bourgat 1977) adds support for using homogenization theory to analyze not only the mechanics of periodic materials, but other processes such as heat conduction and fluid flow as well.

\section{Acknowledgements}

This study was supported by a grant from the National Institutes of Health (RO1 - AR34399).

\section{References}

Accorsi, M. L. (1988): A method for modelling microstructural material discontinuities in a finite element analysis. Int. J. Num. Meth. Eng. 36, 2187-2197

Accorsi, M. L.; Nemat-Nasser, S. (1986): Bounds on the overall elastic and instantaneous elastoplastic moduli of periodic composites. Mech. Materials 5, 209-220

Babuska, I. (1976a): Homogenization approach in engineering. Proc. 2nd Int. Symposium on Comp. Meth. in App. Science and Eng. 137-153. Berlin, Heidelberg, New York: Springer

Babuska, I. (1976b): Solution of interface problems by homogenization. I. SIAM J. Math. Anal. 7,603-634

Babuska, I. (1976c): Solution of interface problems by homogenization. II. SIAM J. Math. Anal. 7, 635-645

Babuska, I. (1976d): Solution of interface problems by homogenization. III. SIAM J. Math. Anal. 7, 923-937

Bakhvalov, N.; Panasenko, G. (1989): Homogenization: averaging processes in periodic media. Dordrecht, The Netherlands: Kluwer Academic Publishers

Beaupre, G.; Hayes, W.(1985): Finite element analysis of a three-dimensional open-celled model for trabecular bone. J. Biomech. Eng. 107, 249-256

Bensoussan, A.; Lions, J.; Papanicolaou, G. (1978): Asymptotic Analysis for Periodic Structures. Amsterdam: North-Holland

Benveniste, Y. (1987): A new approach to the application of Mori-Tanaka's theory in composite materials. Mech. Materials 6, $147-157$

Bourgat, J. (1977): Numerical experiments of the homogenization method for operators with periodic coefficients. In: Dold, A.;

Eckmann, B. (eds): Lecture Notes in Mathematics 704, pp. 330-356. Berlin, Heidelberg, New York: Springer

Budiansky, B. (1965): On the elastic moduli of some heteorogeneous materials. J. Mech. Phys. Solids 13, 223-227

Christenson, R. (1986): Mechanics of low density materials. J. Mech. Phys. Solids 34, 563-578

Cleary, M. P.; Chen, I. W.; Lee, S. M. (1980): Self-consistent techniques for heterogeneous solids. ASCE J. Eng. Mech. 106, $861-887$

Dumontet, H. (1985): Boundary layer stresses in elastic composites. In: Ladeveze, P. (ed): Local Effects in the Analysis of Structures. Amsterdam: Elsevier 215-232

Duvaut, G. (1976): Homogeneization et materiaux composite. In: Ciarlet, P.; Rouseau, M. (eds): Theoretical and Applied Mechanics. Amsterdam: North-Holland

Eshelby, J. D. (1957): The determination of the elastic field of an ellipsoidal inclusion, and related problems. Proc. R. Soc. London A241, 376-396

Fung, Y. (1965): Foundations of Solid Mechanics. Englewood Cliffs: Prentice-Hall

Gibson, L.; Ashby, M. (1982): The mechanics of three-dimensional cellular materials. Proc. R. Soc. London A382, 43-59

Gibson, L.; Ashby, M. (1988): Cellular Solids: Structure and Properties. Oxford: Pergamon Press 
Guedes, J. (1990): Nonlinear computational models for composite materials using homogenization. Ph.D. Dissertation, Ann Arbor: The University of Michigan

Guedes, J.; Kikuchi, N. (1990): Preprocessing and postprocessing for materials based on the homogenization method with adaptive finite element solutions. Com. Mech. App. Meth. Eng. 83, 143-198

Hashin, Z. (1983): Analysis of composite materials-a survey. J. App. Mech. 50, 481-505

Hashin, Z.; Shtrikman, S. (1962): A variational approach to the theory of the elastic behavior of polycrystals. J. Mech. Phys. Solids 10, 343-352

Hashin, Z; Shtrikman, S. (1963): A variational approach to the theory of the elastic behavior of multiphase materials. J. Mech. Phys. Solids 11, 127-140

Hill, R. (1963): Elastic properties of reinforced solids: some theoretical principles. J. Mech. Phys. Solids 11, 357-372

Hill, R. (1965): A self consistent mechanics of composite materials. J. Mech. Phys. Solids 13, 213-222

Huber, A.; Gibson, L. (1988): Anisotropy of foams. J. Mat. Sci. 23, 3031-3040

Iremonger, M. J.; Lawler, J. P. (1980): Relationship between modulus and density for high-density closed cell thermoplastic foams. J. App. Polymer Sci. 25, 809-819

Ishai, O.; Cohen, L. J. (1967): Elastic properties of filled and porous epoxy composites. Int. J. Mech. Sci. 9, 539-546

Iwakuma, T.; Nemat-Nasser, S. (1983): Composites with periodic microstructure. Comput. Struct. 16, 13-19

Kanakkanatt, S. V. (1973): Mechanical anisotropy of open-cell foams. J. Cell. Plast. 9, 50-53

Keller, J. B. (1977): Effective behavior of heterogenous media. Proc. Symposium on Statistical Mechanics and Statistical Methods, Plenum Press, 631-644

Kıkuchi, N. (1986): Finite Element Methods in Mechanics. Cambridge: Cambridge University Press

Kikuchi, N. (in preparation): Variational methods and optimization in mechanics. Englewood Cliffs: Prentice-Hall

Ko, W. (1965): Deformations of foamed elastomers. J. Cell. Plast. 1, 45-50

Kroner, E. (1977): Bounds for effective elastic moduli of disordered materials. J. Mech. Phys. Solids 25, 137-155

Larsen, E. W. (1975): Neutron transport and diffusion in inhomogeneous media. I. J. Math. Phys. 16, 1421-1427

Lederman, J. M. (1971): The prediction of the tensile properties of flexible foams. J. App. Polymer Sci. 15, 693-703

Lene, F.; Leguillon, D. (1982): Homogenized constitutive law for a partially cohesive composite material. Int. J. Solids Struct. $5,443-458$

Lions, J. (1981): Some methods in the Mathematical Analysıs Analysis of Systems and their Control. New York: Gordon and Breach Science Publishers

Lions, J. L. (1980): Asymptotic expansions in perforated media with a periodic structure. Rocky Mountain J. Math. 10, 125-140

Mori, T.; Tanaka, K. (1973): Average stress in matrix and average elastic energy of materials with misfitting inclusions. Acta Metall. 231, 571-574

Mura, T. (1982): Micromechanics of defects in solids. The Hague: Martinus Nijhoff

Murakami, H.; Toledano, A. (1990): A high-order mixture homogenization of bi-laminated composites. J. App. Mech. 57, $388-396$

Murakami, H.; Maewal, A.; Hegemier, G. A. (1981): A mixture theory with a director for linear elastodynamics of periodically laminated media. Int. J. Solids Struct. 17, 155-173

Nemat-Nasser, S.; Taya, M. (1981): On effective moduli of an elastic body containing periodically distributed voids. Quart. J. App. Math. 39, 43-59

Nemat-Nasser, S.; Iwakuma, T.; Hejazi, M. (1982): On composites with periodic structure. Mech. Materials 1, 239-267

Norris, A. N. (1985): A differential scheme for the effective moduli of composites. Mech. Materials 4, 1-16

Norris, A. N. (1989): An examination of the Mori-Tanaka effective medium approximation for multiphase composites. J. App. Mech. 56, 83-88

Patel, M.; Finnie, I. (1970): Structural features and mechanical properties of rigid cellular plastics. J. Mater. 5, 909-932

Reuss, A. (1929): Berechnung der Fliessgrenze von Mischkristallen auf Grund der Plastizitätsbedingung für Einkristalle. Z. Angew. Math. Mech. 9, 49-58

Sanchez-Palencia, E. (1974): Comportements local et macroscopique d'un type de milieux physiques heterogenes. Int. J. Eng. Sci. 12, 331-351

Sanchez-Palencia, E. (1980): Non-Homogeneous Media and Vibration Theory. Berlin, Heidelberg, New York: Springer

Sanchez-Palencia, E. (1987): Boundary layers and edge effects in composites. In: Sanchez-Palencia, E.; Zaoui, A. (eds): Homogenization techniques for composite media, pp. 194-278. Berlin, Heidelberg, New York: Springer

Suquet, P. (1987): Elements of homogenization theory for inelastic solid mechanics. In: Sanchez-Palencia, E.; Zaoui, A. (eds): Homogenization techniques for composite media, pp. 194-278. Berlin, Heidelberg, New York: Springer

Voigt, W. (1889): Über die Beziehung zwischen den beiden Elastizitätskonstanten isotroper Körper, Wied. Ann. 38, 573-587

Walpole, L. J. (1966a): On bounds for the overall elastic moduli of inhomogeneous systems - I. J. Mech. Phys. Solids 14, $151-162$

Walpole, L. J. (1966b): On bounds for the overall elastic moduli of inhomogeneous systems-II. J. Mech. Phys. Solids 14 , 289-301

Warren, W. E.; Kraynik, A. M. (1988): The linear elastic properties of open-cell foams. J. App. Mech. 55, 341-346

Willis, J. R. (1977): Bounds and self-consistent estimates for the overall properties of anisotropic composites. J. Mech. Phys. Solids 25, 185-202 Zhikov, V. V.; Kozlov, S. M.; Oleinik, O. A.; Kha Ten Ngoan (1979): Averaging and G-convergence of differential operators. Rus.
Math. Survey 34, 69-147 